\title{
Fourier analysis on the affine group, quantization and noncompact Connes geometries
}

\author{
Victor Gayral, José M. Gracia-Bondía, and Joseph C. Várilly \\ Dedicated to Orietta Protti, who started it all
}

\begin{abstract}
We find the Stratonovich-Weyl quantizer for the nonunimodular affine group of the line. A noncommutative product of functions on the half-plane, underlying a noncompact spectral triple in the sense of Connes, is obtained from it. The corresponding Wigner functions reproduce the time-frequency distributions of signal processing. The same construction leads to scalar Fourier transformations on the affine group, simplifying and extending the Fourier transformation proposed by Kirillov.
\end{abstract}

PACS numbers. 02.30.Sa, 02.40.Gh.

Mathematics Subject Classification (2000). 43A30, 43A85, 58B34, 81S30.

Keywords. Scalar Fourier transforms, affine symmetry, nonunital spectral triples, Kirillov orbit method, Stratonovich-Weyl quantization.

\section{Contents}

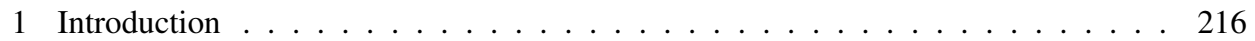

2 The orbit method for the group of affine transformations . . . . . . . . . . . . . 219

3 An unusual special function . . . . . . . . . . . . . . . . . . . 225

4 The Stratonovich-Weyl quantizer for the ' $a x+b$ ' group . . . . . . . . . . . . . 228

5 The Moyal twisted product on the half-plane . . . . . . . . . . . . . . . . . . 234

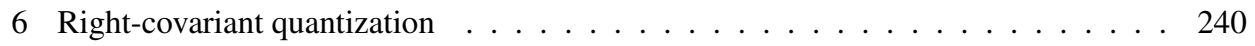

7 Fourier-Moyal transformations on the ' $a x+b$ ' group . . . . . . . . . . . . . . 242

8 Discussion . . . . . . . . . . . . . . . . . . . . . . . . . . . . . . . 249

9 Spectral triples on the half-plane . . . . . . . . . . . . . . . 252

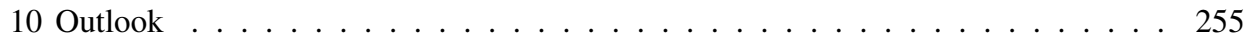

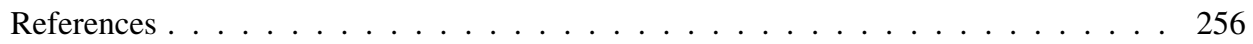




\section{Introduction}

The theory of noncompact spin geometries in the sense of Connes [20], [21] or noncompact spectral triples, broached in [43], was developed in references [67], [38], [40]. The coordinate algebras treated in [67] have locality properties analogous to those of the commutative case, whereas [38], [40] deal with truly noncommutative contexts that are essentially flat, respectively the Moyal $2 n$-planes and some of their generalizations.

As such, the theory remains underdeveloped. This is partly for want of suitable noncommutative noncompact spectral triples. Among the myriads of deformations or "star products", twisted product algebras with the crucial traciality property distinguish themselves in that the classical integral yields a faithful tracial state. This is what made the original (Groenewold-)Moyal product [47], [61], [9] so popular in quantum field theory [32], [78], [72]. Not least, it ensures relevant properties in cyclic cohomology [22].

Let $X$ be a phase space, $\mu$ a convenient measure on it (often the Liouville measure) and $\mathscr{H}$ the Hilbert space associated to $(X, \mu) .{ }^{1}$ Denote by $\delta_{\mu}\left(x, x^{\prime}\right)$ the reproducing kernel for $\mu$. A Stratonovich-Weyl quantizer or tracial quantizer for $(X, \mu, \mathscr{H})$ is an operator-valued distribution $\Omega$ on $X$, with values in the space of selfadjoint operators on $\mathscr{H}$, spanning a weakly dense subset of $\mathscr{B}(\mathscr{H})$, and verifying

$$
\operatorname{Tr} \Omega(x)=1, \quad \operatorname{Tr}\left[\Omega(x) \Omega\left(x^{\prime}\right)\right]=\delta_{\mu}\left(x, x^{\prime}\right) .
$$

Quantizers in this sense, if they exist, are essentially unique. Ownership of a quantizer solves in principle all quantization problems: quantization of a (sufficiently regular) function or "symbol" $a$ on $X$ is effected by

$$
a \mapsto \int_{X} a(x) \Omega(x) d \mu(x)=: Q(a),
$$

and dequantization of an operator $A \in B(\mathscr{H})$ is achieved by

$$
A \mapsto \operatorname{Tr}[\Omega(\cdot) A]=: W_{A}(\cdot) .
$$

Indeed $\Omega$ can just as well be called a dequantizer. It follows that $1_{\mathscr{H}} \mapsto 1$ by dequantization, and also

$$
\operatorname{Tr} Q(a)=\int_{X} a(x) d \mu(x) .
$$

Moreover, since the set $\Omega(X)$ is total, it is clear that

$$
W_{Q(a)}(x)=\operatorname{Tr}\left[\left(\int_{X} a\left(x^{\prime}\right) \Omega\left(x^{\prime}\right) d \mu\left(x^{\prime}\right)\right) \Omega(x)\right]=a(x),
$$

\footnotetext{
${ }^{1}$ This paragraph and the next are excerpted from the report by one of us (JMGB) to the Oberwolfach conference on Dirac Operators and Noncommutative Geometry, in November 2006; see Oberwolfach Rep. 3 (2006), pp. 3151-52.
} 
so $Q$ and $W$ are inverse to one another. In particular, $W_{Q(1)}=1$ says that $1 \mapsto 1 \mathscr{H}$ by quantization. Finally, the following relation holds:

$$
\operatorname{Tr}[Q(a) Q(b)]=\int_{X} a(x) b(x) d \mu(x) .
$$

This is the tracial property.

Most of the interesting cases occur in the context of group actions; that is to say, there is a Lie group $G$ for which $X$ is a symplectic homogeneous $G$-space, with $\mu$ then being a $G$-relatively invariant measure on $X$, and $G$ acts by a (multiplier) unitary irreducible representation $U$ on $\mathscr{H}$. A quantizer for the data set $(X, \mu, \mathscr{H}, G, U)$ satisfies the previous defining equations and is endowed with the covariance property:

$$
U(g) \Omega(x) U^{\dagger}(g)=\Omega(g \triangleright x),
$$

for all $g \in G, x \in X$. Orbits of the coadjoint action of $G$ on its Lie algebra dual $\mathrm{g}^{*}$ and symplectic homogeneous manifolds are essentially the same thing [53], Section 1.4, and in this paper we think of the action denoted by $\triangleright$ above as an instance of the coadjoint action of $G$.

A covariant collection as above, but satisfying only

$$
\int_{X} \Omega(x) d \mu(x)=1_{\mathscr{H}} \quad \text { and } \quad \operatorname{Tr} \Omega(x)=1,
$$

may be called a semitracial quantizer.

Once in possession of the quantizer, one can in principle immediately construct a twisted product that will be normalized (its identity being the constant function 1), hermitian (complex conjugation being the involution), covariant under an appropriate group action, and tracial. We give the details in the body of the paper.

In summary, the "Stratonovich-Weyl" label here refers neither to general deformations nor to star products obtained (roughly speaking) by reduction [43], extension [39] or induction from the original one; but to a restricted category, defined by a precise set of postulates, designed to capture the main trait behind the success of Moyal's formalism for Quantum Mechanics; namely, that quantum and classical expected values should be computed by the same rule. The products thereby obtained are non-formal and analytically controlled. The quest for quantizers in our sense is richly rewarding. In this paper we show by example how the theory of covariant tracial quantizers meshes with, and substantially complements, Kirillov's method of orbits in representation theory. Its main end products are the (scalar) Fourier-Moyal kernels on $\mathrm{g}^{*} \times G$ :

$$
\mathbb{E}(x, g):=\operatorname{Tr}[\Omega(x) U(g)] ; \quad \mathbb{E}^{\bmod }(x, g):=\operatorname{Tr}[\Omega(x) U(g) \sqrt{d}],
$$

with $d$ the formal dimension operator for $U$. There is a good case, that we have made before [31] and we make here again, for these to be the central objects in harmonic 
analysis. For, as soon as a tracial quantizer is available, the abstract Plancherel theorem of group Fourier transform theory becomes a concrete one on the coadjoint orbits. Also, quantizers have an important applied side, with their relations to wavelets in signal processing and to quantum optics.

Before giving the usual guide to the article, we briefly return to noncompact spectral triples. It seems natural to look for noncommutative tracial algebras on the surface of constant negative curvature, whether it be modelled by a hyperboloid in $\mathbb{R}^{3}$, the unit disk, or the Poincaré upper half-plane $\Pi$; we shall focus on the half-plane. Even so, the prescription that the noncommutative coordinate algebras on $\Pi$ carry the full $\operatorname{SL}(2, \mathbb{R})$ symmetry may be too much to ask, because then the first-order condition of [20], [21] for the standard Dirac operator on $\Pi$ cannot be satisfied. This will be shown in Section 9. One can of course inherit the full symmetry and use the alternative Dirac operator; or perhaps "deform" suitably the latter. ${ }^{2}$

Meanwhile we concentrate on the smaller ' $a x+b$ '-type group of symmetry of $\Pi$. As mentioned, there is another compelling motivation for revisiting the StratonovichWeyl quantizers: the progress of photonics [57] allows nowadays a quasi-measurement of the Wigner functions - see [71], [80], [7] as theoretical harbingers. This is calling for investigation of systems with solvable group symmetry; and before tackling them, it helps to visit the case of affine-group symmetry.

In the next section, we remind the reader of the Kirillov method for constructing the unitary irreducible representations (unirreps) of the ' $a x+b$ ' group, in order to make the exposition self-contained. We exhibit the corresponding characters and the Duflo-Moore operator for the nontrivial representations. Section 3 is a modicum of real analysis, eventually needed for establishing the properties of the affine-group quantizers.

Sections 4 to 8 deal with the main issue. As it happens, the literature on wavelets and time-frequency distributions already contains the information needed to extract the quantizers on the half-plane [10], [11]: an impressive example that concrete problem-oriented work can lead to far-reaching conceptual results. A suitable modification of the classical Weyl quantization rule holds. We give the quantizers explicitly and verify the key tracial property in Section 4. In the next section, we find the (left-covariant) twisted product associated to the quantizer, required for spectral triple theory, and investigate its symmetry properties. Section 6 deals with the rightcovariant counterparts for the quantizer and the twisted product. In Section 7, the foregoing illuminates harmonic analysis: scalar Fourier-Moyal transformations are found for the ' $a x+b$ ' group, allowing us to recover the representation characters and to improve on Kirillov's Fourier transformation. The basic results of Fourier analysis, up to and including the Plancherel formula, are shown to hold in our context, for this nonunimodular case. Section 8 connects and compares our formulation with others, including Fronsdal's $\star$-representation program [36] and the approach in a remarkable

\footnotetext{
${ }^{2}$ We thank Pierre Bieliavsky for illuminating discussions of these aspects.
} 
series of recent papers [2], [3], [54] by Ali and coworkers, also inspired by the literature on wavelet transforms. We take the occasion to set the record straight on the matter of Stratonovich-Weyl quantizers.

Section 9 revisits the noncompact spectral triples over the half-plane that have motivated the present work; the first-order property for the Dirac operator can now be established. Section 10 gives pointers for further rapprochement of Connes', Kirillov's and Moyal's paradigms inter alia.

It remains to add that the standards of formality in this paper are about the usual ones in mathematical physics; this saves considerable spacetime. All our arguments are in fact rigorous, as will be shown in [41].

\section{The orbit method for the group of affine transformations}

2.1. The coadjoint orbits. The group Aff of orientation-preserving linear transformations of the real line, or affine group for short, also known as the ' $a x+b$ ' group, is a semidirect product $\mathbb{R}_{+}^{\times} \rtimes \mathbb{R}$, with the handy matrix realization

$$
\text { Aff } \equiv\left\{\left(\begin{array}{ll}
a & b \\
0 & 1
\end{array}\right): a>0, b \in \mathbb{R}\right\} .
$$

Its Lie algebra is realized by the matrices

$$
\text { aff }:=\left\{X=\left(\begin{array}{cc}
u & v \\
0 & 0
\end{array}\right):(u, v) \in \mathbb{R}^{2}\right\}, \quad X=u X_{1}+v X_{2},
$$

with commutation relation $\left[X_{1}, X_{2}\right]=X_{2}$. The group is solvable, since its Lie algebra has the ideal $\mathbb{R} X_{2}=$ [aff, aff] with aff / $\mathbb{R} X_{2}$ abelian. Recall that a group $G$ and its tangent Lie algebra $g$ are called exponential if the map exp: $g \rightarrow G$ is a surjective diffeomorphism. Note that

$$
\operatorname{ad} X=\left(\begin{array}{cc}
0 & -v \\
0 & u
\end{array}\right), \quad \text { with eigenvalues } 0, u \text {. }
$$

By an old result of Dixmier [25], since these eigenvalues are not (nonzero) purely imaginary, the group is exponential. Of course, this can be seen already from (2.1), since

$$
g(u, v):=\exp \left(u X_{1}+v X_{2}\right)=\left(\begin{array}{cc}
e^{u} & v\left(e^{u}-1\right) / u \\
0 & 1
\end{array}\right) .
$$

Since $\operatorname{tr}(\operatorname{ad} X) \neq 0$ in general, the group is not unimodular. Indeed, the right and left Haar measures $d_{r} g$ and $d_{l} g$ on Aff are respectively given by

$$
\begin{aligned}
& d_{r}(\exp X)=\operatorname{det}\left(\frac{e^{\mathrm{ad} X}-1}{\operatorname{ad} X}\right) d X=\frac{d u d v}{\lambda(-u)}=\frac{d a d b}{a}, \\
& d_{l}(\exp X)=\operatorname{det}\left(\frac{1-e^{-\mathrm{ad} X}}{\operatorname{ad} X}\right) d X=\frac{d u d v}{\lambda(u)}=\frac{d a d b}{a^{2}},
\end{aligned}
$$


where $d X=d u d v$ and

$$
\lambda(t):=\frac{t e^{t}}{e^{t}-1}=\frac{t}{1-e^{-t}}=\frac{e^{t / 2}}{\operatorname{sinch}(t / 2)}=e^{t / 2} \Gamma\left(1+\frac{t}{2 \pi i}\right) \Gamma\left(1-\frac{t}{2 \pi i}\right),
$$

with $\operatorname{sinch} t:=(\sinh t) / t$ (and $\operatorname{sinch} 0:=1)$, a well-known nonvanishing even function (so named, by analogy with the sinus cardinalis of sampling theory). Note that the right Haar measure on Aff is the product of the Haar measures of $\mathbb{R}_{+}^{\times}$and $\mathbb{R}$; this is a general property for semidirect products. Neither the left nor right Haar measure coincides with the measure induced on Aff by the Lebesgue measure on aff. The last equalities on the right-hand sides of (2.3) follow from (2.2). Therefore the modular function $\Delta(g):=d_{l} g / d_{r} g$ is given by $1 / a$. We also note for future use the product of the densities (normalized at 0 ) of the Haar measures with respect to the Lebesgue measure,

$$
j_{l}(X) j_{r}(X):=\frac{d_{l}(\exp X)}{d X} \frac{d_{r}(\exp X)}{d X}=\operatorname{sinch}^{2}(u / 2) .
$$

The adjoint action of Aff on aff, and the contragredient coadjoint action on aff*, are respectively given by

$$
\operatorname{Ad} g(X):=g X g^{-1}=\left(\begin{array}{cc}
u & a v-b u \\
0 & 0
\end{array}\right) ; \quad\langle g \triangleright F, X\rangle:=\left\langle F, \operatorname{Ad} g^{-1}(X)\right\rangle,
$$

for $X \in$ aff, $F \in$ aff $^{*}$; we generally use the letter $F$ for points in coalgebras $\mathrm{g}^{*}$. Also, aff* can be realized by matrices

$$
F=(x, y):=\left\{\left(\begin{array}{ll}
x & 0 \\
y & 0
\end{array}\right):(x, y) \in \mathbb{R}^{2}\right\} .
$$

Thus $\langle F, X\rangle=\operatorname{tr}(F X)=u x+v y$, and the coadjoint action is given by

$$
(x, y) \mapsto g \triangleright(x, y) \equiv \operatorname{Coad} g(x, y)=\left(x+\frac{b y}{a}, \frac{y}{a}\right) .
$$

The orbits of this action are two open half-planes, plus an axis of fixed points. Indeed, if we choose any point $F=(x, 0)$, then the whole group leaves it invariant, whereas all the other points of aff ${ }^{*}$ are found in the orbits of $F=(0,+1)$ and of $F=(0,-1)$. The isotropy group in the last two cases is trivial, and both orbits, which we denote by $\mathcal{O}_{ \pm}$, are diffeomorphic to the group itself. We think of $\mathcal{O}_{ \pm}$as Poincaré half-planes, respectively $\Pi$ and $-\Pi$, adopting complex-variable notation when convenient.

We can describe these "solvmanifolds" by group parameters. If

$$
z(g):=g \triangleright \pm i=( \pm b / a, \pm 1 / a), \quad \text { with inverse } z \mapsto g_{z}=( \pm 1 / y, x / y),
$$

then of course $g \triangleright z\left(g^{\prime}\right)=z\left(g g^{\prime}\right)$, for $g, g^{\prime} \in$ Aff. One can employ this to transfer the group operation onto the orbit by $z(g) \cdot z\left(g^{\prime}\right):=z\left(g g^{\prime}\right)$. Explicitly,

$$
(x+i y) \cdot\left(x^{\prime}+i y^{\prime}\right)=x^{\prime} \pm x y^{\prime} \pm i y y^{\prime} .
$$


Reciprocally, $z \mapsto g_{z}$ is an isomorphism, namely $g_{z} g_{z^{\prime}}=g_{z} \cdot z^{\prime}$.

The invariant symplectic forms $\omega_{ \pm}$on $\mathcal{O}_{ \pm}$are exact in the present case, being clearly given by $\omega_{ \pm}(z)=d x d y /|y|$; note that $\omega_{ \pm}(z(g))=d_{l} g$. Darboux coordinates are $q=x / y, p=|y|$.

In general, we say that a subalgebra $\mathfrak{h} \subseteq \mathfrak{g}$ is subordinate to $F \in \mathfrak{g}^{*}$ if $\left.F\right|_{[\mathfrak{h}, \mathfrak{h}]}=0$ and the map $X \mapsto\langle F, X\rangle$ is a one-dimensional representation of $\mathfrak{h}$. The entire Lie algebra aff is subordinate to each $(x, 0)$. Any one-dimensional subalgebra of aff is subordinate to $(0,1)$ or to $(0,-1)$, but only the ideal [aff, aff] is Pukánszky, which means that $F+\mathfrak{h}^{\perp} \subseteq \mathcal{O}_{F}$ : indeed, $(x, 0)+(0,0)=(x, 0)$ and $(0, \pm 1)+\left(x^{\prime}, 0\right) \in \mathcal{O}_{ \pm}$.

2.2. The Kirillov map and the unirreps. The Kirillov theory asserts the existence of

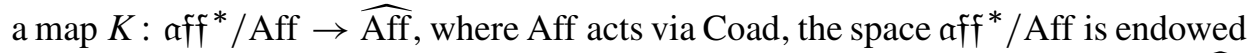
with the (non-Hausdorff, not even $T_{1}$ ) quotient topology and the unitary dual $\widehat{\text { Aff }}$ with its standard Fell topology, determined by the hull-kernel topology on the set of primitive ideals of $C^{*}(G)$ - this matter is well explained in [26], Chapter 3, or [29], Chapter 7. For exponential groups, $K$ has been proved by Leptin and Ludwig [55] to be bijective and bicontinuous. (For the similar correspondence between $\mathrm{g}^{*} / G$ and the set of primitive ideals of the enveloping algebra $U(g)$, we refer to [58].) It is known that all unirreps for exponential groups are monomial, that is, induced by an abelian character of some closed subgroup $H$. If $H$ is the closed subgroup generated by $\mathfrak{h}$ subordinate to $F$, the Pukánszky condition guarantees that the induced representations

$$
K\left[\mathcal{O}_{F}\right](\exp X):=\operatorname{Ind}_{H}^{\mathrm{Aff}} U_{F, H}(\exp X)=\operatorname{Ind}_{H}^{\mathrm{Aff}} e^{2 \pi i\langle F, X\rangle}
$$

are indeed irreducible. In the present case,

$$
U_{(x, 0), \operatorname{Aff}}(\exp X)=e^{2 \pi i x u} \quad \text { and } \quad U_{ \pm, \exp \left(\mathbb{R} X_{2}\right)}\left(\exp b X_{2}\right)=e^{ \pm 2 \pi i b} .
$$

So we obtain in the first place the unitary one-dimensional representations $(a, b) \mapsto$ $a^{2 \pi i x}$ of Aff; observe that $K\left[\mathcal{O}_{(0,0)}\right]$ is the trivial representation.

Now denote $U_{ \pm}:=K\left[\mathcal{O}_{ \pm}\right]$. The Kirillov scheme "predicts" (this is only of heuristic value) that the "functional dimension" of $U_{ \pm}$is $\frac{1}{2} \operatorname{dim} \mathcal{O}_{ \pm}=1$; that is, $U_{ \pm}$ can be realized on spaces of functions of one variable, in such a way that the smooth vectors are smooth functions and the enveloping algebra $U$ (aff) acts by differential operators. The actual induction process leads us to consider the space of functions $f$ on Aff such that

$$
f(a, b)=e^{ \pm 2 \pi i b} \psi(a)
$$

and then, necessarily,

$$
U_{ \pm}(a, b) f\left(a^{\prime}, b^{\prime}\right)=f\left(a a^{\prime}, b a^{\prime}+b^{\prime}\right) \quad \text { or } \quad U_{ \pm}(a, b) \psi\left(a^{\prime}\right)=e^{ \pm 2 \pi i b a^{\prime}} \psi\left(a a^{\prime}\right) .
$$

That is, we may settle on

$$
U_{ \pm}(a, b) \psi(r)=e^{2 \pi i b r} \psi(a r)=U_{ \pm}(1, b) U_{ \pm}(a, 0) \psi(r),
$$


with $r>0$ for $U_{+}$and $r<0$ for $U_{-}$. The $U_{ \pm}$preserve the space of smooth functions on the semiaxis, vanishing in some neighbourhood of $r=0$, and are unitary on the Hilbert spaces $\mathcal{K}_{ \pm}:=L^{2}\left((0, \pm \infty), r^{-1} d r\right)$. Observe that $U_{+}$and $U_{-}$are mutually dual; this is a general property for $K[\mathcal{O}]$ and $K[-\mathcal{O}]$. (Needless to say, the process of going from $\mathcal{O}$ to a putative $K[\mathcal{O}]$ is not always so smooth; experience points to the importance of $\mathcal{O}$ being spin and of its twisted Dirac operator to construct $K[\mathcal{O}]$ - see [23] in this respect.)

The selfadjoint infinitesimal generators for the unirreps are given by

$$
\begin{aligned}
& U_{ \pm}\left(X_{1}\right) \psi(r)=-\left.i \frac{d}{d u}\right|_{u=0} U_{ \pm}(\exp (u, 0)) \psi(r)=-i r \psi^{\prime}(r) ; \\
& U_{ \pm}\left(X_{2}\right) \psi(r)=-\left.i \frac{d}{d v}\right|_{v=0} U_{ \pm}(\exp (0, v)) \psi(r)=2 \pi r \psi(r) .
\end{aligned}
$$

We denote the generators $U_{ \pm}\left(X_{1}\right), U_{ \pm}\left(X_{2}\right)$ by $2 \pi \hat{\beta}_{ \pm}, 2 \pi \hat{f}_{ \pm}$respectively. Note $2 \pi i\left[\hat{\beta}_{ \pm}, \hat{f}_{ \pm}\right]=\hat{f}_{ \pm}$.

Interesting (improper) denizens of $\mathcal{K}_{ \pm}$are the "plane waves" or eigenfunctions of $\hat{\beta}_{ \pm}$. They are given by $\psi_{\beta}(r):=r^{ \pm 2 \pi i \beta}$, for $\beta$ real, and constitute a (generalized) orthonormal and complete set. Complete orthonormal sets within $\mathcal{K}_{ \pm}$are also known, but here we shall not use them.

2.3. Characters of the unirreps and Kirillov's Fourier transform. Any operator $A$ on $\mathcal{K}_{ \pm}$determines an integral kernel, with suitable genuflections to rigour:

$$
A \psi(r)=: \int_{\mathbb{R}_{ \pm}} A(r, s) \psi(s) \frac{d s}{s},
$$

so that the operator kernel of $U_{ \pm}(a, b)$ is

$$
U_{ \pm}(a, b ; r, s)=e^{2 \pi i b r} s \delta(s-a r) .
$$

Let $f \in \mathscr{D}($ Aff $) \equiv C_{c}^{\infty}$ (Aff). Following Kirillov [53], Section 4.1, we define the associated operators $U_{ \pm}(f)$ on $\mathcal{K}_{ \pm}$by

$$
\begin{aligned}
U_{ \pm}(f) \psi(r): & =\int_{0}^{\infty} \int_{-\infty}^{\infty} f(a, b) e^{2 \pi i b r} \psi(a r) \frac{d a d b}{a} \\
& =\int_{0}^{ \pm \infty} \int_{-\infty}^{\infty} f(s / r, b) e^{2 \pi i b r} \psi(s) \frac{d s d b}{s}
\end{aligned}
$$

with kernels

$$
U_{ \pm}(f ; r, s)=\int_{-\infty}^{\infty} f(s / r, b) e^{2 \pi i b r} d b,
$$

where $r>0, s>0$ or $r<0, s<0$, respectively. One would naïvely expect that $U_{ \pm}(f)$ be nuclear, for $f$ a test function. This is not the case unless $f$ is of zero 
mean with respect to the second variable; otherwise $U_{ \pm}(f)$ is not even compact [52]: $C^{*}$ (Aff) is not liminaire. Assume, however, that this requirement holds; then the traces are given by

$$
\operatorname{Tr} U_{ \pm}(f)=\int_{-\infty}^{\infty} \int_{\mathbb{R}_{ \pm}^{\times}} f(1, b) e^{2 \pi i b r} \frac{d b d r}{r}
$$

for $r>0$ or $r<0$, respectively. By definition, the (generalized) characters $\chi_{ \pm}$of $U_{ \pm}$are the functionals such that

$$
\chi_{ \pm}(f)=\operatorname{Tr} U_{ \pm}(f)
$$

Let $\tilde{f}:=f \circ$ exp. We see that $\chi_{ \pm}(f)$ only depends on the values of $\tilde{f}$ on [aff, aff]; this is a particular instance of a property established by Duflo [27]. We can say that

$$
\chi_{ \pm}(a, b)=\delta(a-1) \int_{\mathbb{R}_{ \pm}^{\times}} e^{2 \pi i b r} \frac{d r}{r}
$$

or $F_{2} \chi_{ \pm}(a, r)=\frac{\theta( \pm r)}{r} \delta(a-1)$, where $\theta$ is the Heaviside function, with the obvious caveats for good definition in these "ultraviolet divergent" expressions.

For simply connected nilpotent groups, which are unimodular and whose Haar measure is the Lebesgue measure in exponential coordinates, Kirillov postulated and showed the existence of a unitary transformation matching $L^{2}(G)$ with $L^{2}\left(g^{*}\right)$, here denoted $\mathbb{F}_{\mathrm{K}}$, of the form

$$
\mathbb{F}_{\mathrm{K}}[f](F):=\int_{\mathfrak{g}} \tilde{f}(X) e^{2 \pi i\langle F, X\rangle} d X,
$$

and he established the formula

$$
\chi_{K[\mathcal{O}]}(\exp X)=\int_{\mathcal{O}} e^{2 \pi i\langle F, X\rangle+\omega},
$$

where $\omega$ is the invariant symplectic form on $\mathcal{O}$, so that

$$
\operatorname{Tr} K[\mathcal{O}](f):=\int_{\mathfrak{g}} f(\exp X) \chi_{K[\mathcal{O}]}(\exp X) d X=\int_{\mathcal{O}} \mathbb{F}_{\mathrm{K}}[f](F) d \mu_{\omega}(F)
$$

Here $\mu_{\omega}$ is the Liouville measure on the orbit, given by $\omega^{\frac{1}{2} \operatorname{dim} \mathcal{O}} /\left(\frac{1}{2} \operatorname{dim} \mathcal{O}\right)$ ! ; also, (2.10) clearly means that $\mathbb{F}_{K}\left[\chi_{K[\mathcal{O}]}\right]$ - a tempered distribution defined on $g$ by transposition, transported to $G$ by the exponential map - coincides precisely with that measure. This was one of the earliest triumphs of the method of orbits. The similarity of (2.9) with the relation between the classical and quantum partition functions has been pointed out and exploited before: see [70]. 
For general solvable groups, this will not do. Part of the problem is that the orbit is open and the invariant symplectic structure is singular at the boundary. Also, it is unclear which measure to use on $g$, in the nonunimodular case. Kirillov suggests that the recipe (2.9) be replaced by a weighted version:

$$
\chi_{K[\mathcal{O}]}(\exp X)=\frac{1}{q(X)} \int_{\mathcal{O}} e^{2 \pi i\langle F, X\rangle+\omega}
$$

and for $q$ he chooses $\left(j_{l} j_{r}\right)^{1 / 4}$. In view of (2.5), for Aff this leads to

$$
\begin{aligned}
& \mathbb{F}_{\mathrm{K}}[f](z) \\
& =\iint_{\mathbb{R}^{2}} \exp \{2 \pi i(u x+v y)\} f\left(e^{u}, \frac{v}{\lambda(-u)}\right) \frac{d u d v}{\sqrt{\operatorname{sinch} \frac{u}{2}}} \\
& =\iint_{\text {Aff }} \exp \{2 \pi i(x \log a+y b \lambda(-\log a))\} f(a, b) \lambda^{1 / 4}(\log a) \lambda^{5 / 4}(-\log a) \frac{d a d b}{a},
\end{aligned}
$$

for $z \in a f f^{*}$. It is necessary and sufficient that $\mathbb{F}_{K}[f]$ go to zero on the boundary of the orbits for $K\left[\mathcal{O}_{ \pm}\right](f)$ to be nuclear. Then (2.10) is still valid. This is scarcely surprising since, as pointed out earlier, only the value of $\lambda$ at 0 enters the calculation. However, the last formula is certainly not pretty.

Yet another a priori Fourier map is defined in [3] by a formula of the type

$$
\mathbb{F}_{\mathrm{AFK}}[f](F)=\sqrt{\xi_{j}(F)} \int_{\mathrm{g}^{*}} \exp \{2 \pi i\langle F, X\rangle\} f(\exp X) \sqrt{m(X)} d X .
$$

We explain their notation: $j$ is an index parametrizing the orbits; $\xi_{j}=d F / d \omega_{j}$; and $m(X)=d_{l}(\exp X) / d X$. Here, for $y \neq 0$, we take $j \in\{ \pm\} ; \xi_{ \pm}(z)=|y|$; and $m(u, v)=1 / \lambda(u)$. Hence

$$
\mathbb{F}_{\mathrm{AFK}}[f](z)=\sqrt{|y|} \iint_{\mathbb{R}^{2}} \exp \{2 \pi i(u x+v y)\} f\left(e^{u}, v / \lambda(-u)\right) \frac{1}{\sqrt{\lambda(u)}} d u d v .
$$

By construction, this is an isometry between $L^{2}$ (Aff, $d_{l} g$ ) and $L^{2}\left(\right.$ aff $\left.{ }^{*}, d \omega_{+} \cup d \omega_{-}\right)$. However, it does not give the character.

In this paper we introduce two (left and right) powerful alternative transforms to Kirillov's Fourier map, that likewise recover the character, and are closely related to $\mathbb{F}_{\mathrm{AFK}}$.

2.4. The Duflo-Moore operators. The decomposition of the regular representation of Aff, defined as usual by

$$
\Lambda(g) f\left(g^{\prime}\right):=f\left(g^{-1} g^{\prime}\right)
$$


is well known for the affine group: $\Lambda$ decomposes into a continuous direct sum of representations equivalent to $U_{+} \oplus U_{-}$. More concretely, the Plancherel measure is in our case just the counting measure on the two-element set $\left\{U_{ \pm}\right\}$, and there is a unitary map $P$, the Plancherel transform:

$$
P: L^{2}(G) \rightarrow \operatorname{HS}\left(\mathcal{K}_{+}\right) \oplus \operatorname{HS}\left(\mathcal{K}_{-}\right),
$$

with $\operatorname{HS}\left(\mathcal{K}_{ \pm}\right)$denoting the Hilbert algebras of Hilbert-Schmidt operators on $\mathcal{K}_{ \pm}$, and $P$ given by

$$
P f:=U_{+}(f) d_{+}^{1 / 2} \oplus U_{-}(f) d_{-}^{1 / 2},
$$

where the $d_{ \pm}$are positive operators on $\mathcal{K}_{ \pm}$with densely defined inverses, determined (up to a positive constant) by the semi-invariance relation:

$$
U_{ \pm}(g) d_{ \pm} U_{ \pm}^{\dagger}(g)=\Delta^{-1}(g) d_{ \pm}
$$

For a general proof of this uniqueness for $G$ not unimodular, see [28] and also [4]. Because the $U_{ \pm}$are induced from the subgroup $\{1, b\}$ belonging to the kernel of $\Delta$, it is easily seen that

$$
d_{ \pm} \psi(r)=|r| \psi(r)
$$

where we have chosen a convenient normalization. These $d_{ \pm}$are the formal dimension operators as originally defined by Duflo and Moore in [28], although later authors use the phrase "Duflo-Moore operators" for $d_{ \pm}^{-1 / 2}$ instead. For their theory, one may consult [28] and also its excellent precursor [74]. The remarkable thing is that the operators $U_{ \pm}(f) d_{ \pm}^{1 / 2}$ (or rather, their closures) are Hilbert-Schmidt whenever $f$ belongs to $L^{2}(G)$ - actually, our treatment of the harmonic analysis on Aff in the long Section 7 amounts to an indirect proof of this fact. Then (2.14) holds, and moreover

$$
P(\Lambda(g) f)=\left(U_{+} \oplus U_{-}\right)(g) P f .
$$

Also, for $f$ in a suitable dense subspace of $L^{2}(G)$, the operators $U(f) d_{ \pm}$are nuclear.

\section{An unusual special function}

Before we plunge into calculating the quantizer, it is convenient to perform a few exercises in real analysis, to be rewarded with later simplification.

We begin with the function $\lambda$ of (2.4). Note that $\lambda(0)=1$ and $\lambda(t)>0$ for all $t \in \mathbb{R}$, that $\lambda(t) \downarrow 0$ as $t \rightarrow-\infty$, and that $\lambda(t) \sim t$ as $t \rightarrow+\infty$ (see Figure 1). It is easy to see that

$$
\begin{aligned}
\lambda(-t) & =e^{-t} \lambda(t), \\
\lambda(t)-\lambda(-t) & =t .
\end{aligned}
$$




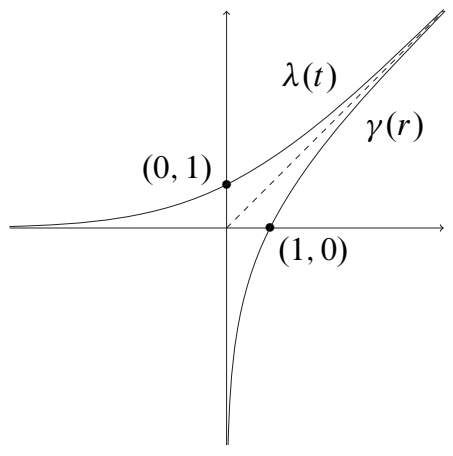

Figure 1. The function $\lambda$ and its inverse function $\gamma$.

These functional equations determine $\lambda$ uniquely. It is an analytic function for $|t|<2 \pi$, with expansion

$$
\lambda(t)=\sum_{n \geq 0}(-1)^{n} B_{n} \frac{t^{n}}{n !} ; \quad \text { thus } \quad \lambda(-t)=\sum_{n \geq 0} B_{n} \frac{t^{n}}{n !},
$$

where the $B_{n}$ are the well-known Bernoulli numbers.

The derivative of $\lambda(t)$ is

$$
\begin{aligned}
\lambda^{\prime}(t) & =\left(1-e^{-t}\right)^{-1}-t e^{-t}\left(1-e^{-t}\right)^{-2}=\frac{\lambda(t)}{t}-\frac{\lambda(t) \lambda(-t)}{t} \\
& =\frac{\lambda(t)}{t}(1-\lambda(-t))=\frac{\lambda(t)}{t}(1+t-\lambda(t)) .
\end{aligned}
$$

Thus $\lambda(t)$ is strictly increasing on $\mathbb{R}$. Since (3.1b) entails $\lambda^{\prime}(t)+\lambda^{\prime}(-t)=1$, we see that $\lambda^{\prime}(0)=\frac{1}{2}$. A brief calculation shows that $\lambda^{\prime \prime}(t)>0$ for $t>0$; since $\lambda^{\prime \prime}(t)=\lambda^{\prime \prime}(-t)$, it follows that $\lambda^{\prime}(t)$ is increasing (i.e., $\lambda(t)$ is convex), with $\lambda^{\prime}(t) \uparrow 1$ as $t \rightarrow+\infty$, and therefore $\lambda^{\prime}(t)<1$ for all $t$.

Next, let $\gamma(r)$ denote the inverse function for $\lambda: \gamma(r)=t$ when $r=\lambda(t)$. It is defined for $r>0$, is strictly increasing with $\gamma(1)=0$ and $\gamma^{\prime}(1)=2$, and $\gamma(r) \downarrow-\infty$ as $r \downarrow 0$, while $\gamma(r) \sim r$ as $r \uparrow+\infty$. All that is evident on reflecting the graph of $r=\lambda(t)$ about the main diagonal $r=t$. Of course, the chain rule and (3.2) give

$$
\gamma^{\prime}(r)=\frac{1}{\lambda^{\prime}(\gamma(r))}=\frac{\gamma(r)}{r(1-r+\gamma(r))} .
$$

In particular, $\gamma^{\prime}(r)>1$ for all $r>0$.

The special function worthy of our attention is

$$
\sigma(r):=r-\gamma(r), \quad \text { for } \quad r>0 .
$$


For $|r|<1$ we obtain, by [30, Section 2.1] for instance,

and

$$
\gamma(1+r)=2 r-\frac{2}{3} r^{2}+\frac{4}{9} r^{3}-\frac{44}{135} r^{4}+\frac{104}{405} r^{5}+\cdots
$$

$$
\sigma(1+r)=1-r+\frac{2}{3} r^{2}-\frac{4}{9} r^{3}+\frac{44}{135} r^{4}-\frac{104}{405} r^{5}+\cdots
$$

Write $s=\lambda(-t)$ and $r=\lambda(t)$. Then relation (3.1b) shows that

$$
s=\sigma(r) \Longleftrightarrow r=\sigma(s),
$$

or, not to put too fine a point on it, $\sigma(\sigma(r))=r$, that is, $\sigma$ is self-inverse.

Note, too, that $\sigma(r)=r e^{-\gamma(r)}$, in view of relation (3.1a). Also, $\sigma(1)=1$ and $\sigma^{\prime}(1)=-1$, as expected since the graph of $\sigma$ must be invariant on reflection in the main diagonal. Now $\sigma^{\prime}(r)=1-\gamma^{\prime}(r)<0$ always, so that $\sigma$ is a strictly decreasing function. Moreover, $\sigma(r) \sim-\gamma(r)$ as $r \downarrow 0$, while $\sigma(r) \sim r e^{-r}$ as $r \rightarrow+\infty$ : the graph of $\sigma$ is exponentially asymptotic to both axes in the first quadrant. Note, as well, from (3.3):

$$
\gamma(r)=-\gamma(\sigma(r)) ; \quad \gamma^{\prime}(r)=-\gamma^{\prime}(\sigma(r)) \sigma^{\prime}(r) .
$$

We remark that $\sigma^{\prime}(r)=e^{-\gamma(r)}\left(1-r \gamma^{\prime}(r)\right)=\sigma(r)\left(r^{-1}-\gamma^{\prime}(r)\right)$; writing $s=\sigma(r)$ gives

$$
s-\sigma^{\prime}(\sigma(s)) \sigma(s)=s-s\left(\frac{1}{\sigma(s)}-\gamma^{\prime}(\sigma(s))\right) \sigma(s)=s \sigma(s) \gamma^{\prime}(\sigma(s)) .
$$

It is also useful for our purposes to dilate $\gamma$ and $\sigma$ by a factor $y>0$, as follows:

$$
\gamma_{y}(r):=y \gamma(r / y), \quad \sigma_{y}(r):=y \sigma(r / y) .
$$

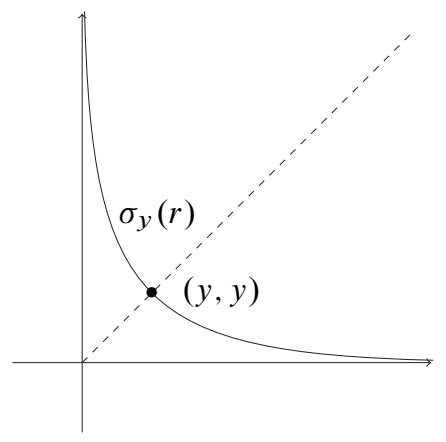

Figure 2. The self-inverse function $\sigma_{y}$ for $y>0$. 
It is immediate that $\sigma_{y}\left(\sigma_{y}(r)\right)=r$, so that $\sigma_{y}$ also has a graph that is unchanged on reflection in the diagonal, except that it now crosses the diagonal at $(y, y)$ : see Figure 2. Moreover, the previous relations between $\gamma$ and $\sigma$ become

$$
\begin{aligned}
\sigma_{y}(r) & =r-\gamma_{y}(r), & \gamma_{y}\left(\sigma_{y}(r)\right) & =-\gamma_{y}(r), \\
\sigma_{y}^{\prime}(r) & =1-\gamma_{y}^{\prime}(r), & \gamma_{y}^{\prime}\left(\sigma_{y}(r)\right) \sigma_{y}^{\prime}(r) & =-\gamma_{y}^{\prime}(r) .
\end{aligned}
$$

\section{The Stratonovich-Weyl quantizer for the ' $a x+b$ ' group}

4.1. Weyl quantization for the half-plane. Pre-existing work on image processing [10], [11] points the way towards the quantizer: the transformation from "density matrices" to "Wigner functions" considered in these papers respects reality, covariance and "unitarity", which is equivalent to traciality. Consider the Weyl operator,

$$
W_{ \pm}(u, v):=\exp \left\{2 \pi i\left(u \hat{\beta}_{ \pm}+v \hat{f}_{ \pm}\right)\right\}=U_{ \pm}\left(e^{u}, v\left(e^{u}-1\right) / u\right)=U_{ \pm}\left(e^{u}, v e^{u} / \lambda(u)\right)
$$

Our candidate quantizer is

$$
\Omega_{ \pm}(x+i y):=|y| \iint_{\mathbb{R}^{2}} \exp \{-2 \pi i(u x+v y)\} W_{ \pm}(u, v) d u d v
$$

It can hardly be simpler! Aside from the factor $|y|$, that compensates for the measures used on $\mathcal{O}_{ \pm}$, it means that the familiar definition from Quantum Mechanics works. The recipe is actually imposed on us by the heuristic rule that $\Omega_{ \pm}\left(F_{0}\right)$ should be the quantization of $\delta\left(F-F_{0}\right)$, or the equivalent remark in [11] that the quantization of a plane wave should be given by the Weyl operator. It stands to reason that the Weyl operator will play an essential role in an exponential group; one may treat (4.1) as an Ansatz, and simply prove that it gives the Stratonovich-Weyl quantizer by verifying all the required properties.

We carry out this verification for the upper half-plane $\Pi$. For $-\Pi$ the argument is identical. It is no longer worth the trouble to keep always the subscripts \pm , so we mostly drop them.

4.2. Identification of the basic "parity" operators. The claim is that we can associate to each symbol $f(z)$ on $\Pi$ an operator $A$ on the representation space $\mathcal{K}_{+} \equiv \mathcal{K}$, and vice versa, by

$$
A=\int_{\Pi} \Omega(z) f(z) d \omega(z)=: Q(f), \quad f(z)=\operatorname{Tr}(\Omega(z) A),
$$

with the properties required in the Introduction. The quantizer $\Omega(z)$ remains to be determined. 
With the machinery assembled in the previous section, the computation of $\Omega$ is straightforward. First of all,

$$
W(u, v) \psi(r)=e^{2 \pi i v e^{u} r / \lambda(u)} \psi\left(e^{u} r\right),
$$

with operator kernel

$$
W(u, v ; r, s)=e^{2 \pi i v s / \lambda(u)} s \delta\left(s-e^{u} r\right) .
$$

The right-hand side of (4.1), applied to $\psi \in \mathscr{D}(0, \infty)$, yields

$$
\begin{aligned}
\Omega(z) \psi(r) & =y \iint_{\mathbb{R}^{2}} e^{-2 \pi i\left(u x+v y-e^{u} r v / \lambda(u)\right)} \psi\left(e^{u} r\right) d u d v \\
& =y \int_{\mathbb{R}} e^{-2 \pi i u x} \delta\left(y-r e^{u} / \lambda(u)\right) \psi\left(e^{u} r\right) d u \\
& =\frac{r}{y} \gamma_{y}^{\prime}(r) e^{2 \pi i x \gamma_{y}(r) / y} \psi\left(\sigma_{y}(r)\right) .
\end{aligned}
$$

For this, consider the diffeomorphism $u \mapsto r / \lambda(-u)=w$, whose inverse mapping is $u=-\gamma(r / w)$ with Jacobian $J(u ; w)=r w^{-2} \gamma^{\prime}(r / w)$. The general formula [79], Chapter 1:

$$
\langle T(w(u)), \psi(u)\rangle=\langle T(w), \psi(u(w))|J(u ; w)|\rangle
$$

in the present case easily gives

$$
\begin{aligned}
\int_{\mathbb{R}} \delta(y & \left.-r e^{u} / \lambda(u)\right) e^{-2 \pi i u x} \psi\left(e^{u} r\right) d u \\
& =\int_{\mathbb{R}} \delta(y-w) e^{2 \pi i x \gamma(r / w)} \psi\left(r e^{-\gamma(r / w)}\right) r w^{-2} \gamma^{\prime}(r / w) d w
\end{aligned}
$$

yielding (4.3). Similar computations will reappear throughout this paper; often we shall just omit them. In particular, we have obtained

$$
\Omega(i) \psi(r)=r \gamma^{\prime}(r) \psi(\sigma(r)) .
$$

A remarkable event has occurred. For the ordinary Moyal family, the "mother" operator in the Schrödinger representation is known to be essentially the (GrossmannRoyer) parity operator. Matters are more involved here, but still $\Omega(i)$ is basically given by a kind of reflection; to wit, the involutive $\sigma$ function.

In view of (2.7), covariance requires $\Omega(z)=U\left(g_{z}\right) \Omega(i) U^{\dagger}\left(g_{z}\right)$, where $U\left(g_{z}\right)=$ $U(1 / y, x / y)$ with adjoint operator $U^{\dagger}\left(g_{z}\right)=U(y,-x)$. We leave this direct verification as an exercise.

The kernel of $\Omega(z)$ is given by

$$
\Omega(z ; r, s)=\frac{r s}{y} \gamma_{y}^{\prime}(r) e^{2 \pi i x(r-s) / y} \delta\left(s-\sigma_{y}(r)\right) .
$$


An alternative form is

$$
\begin{aligned}
\Omega(z ; r, s) & =y e^{2 \pi i x \log (r / s)} \delta\left(y-\frac{r}{\lambda(\log (r / s))}\right) \\
& =y e^{2 \pi i x \log (r / s)} \delta\left(y-\frac{r-s}{\log (r / s)}\right) .
\end{aligned}
$$

For the normalization property the operators $\Omega(z)$ must be of trace 1 , in the distributional sense. We check this by computing

$$
\operatorname{Tr} \Omega(i)=\int_{0}^{\infty} \Omega(i ; r, r) \frac{d r}{r}=\int_{0}^{\infty} r \gamma^{\prime}(r) \delta(\gamma(r)) d r=1 .
$$

The normalization $\operatorname{Tr} \Omega(z)=1$ is then automatic, since the $U\left(g_{z}\right)$ are unitary. It can be proved that the operators $Q(f)$, for $f$ any test function on $\Pi$, are nuclear [41].

4.3. Selfadjointness of the quantizer operators. Note that the $\Omega(z)$ are unbounded operators. They are defined at least on $\mathscr{D}(0, \infty)$. Indeed, since $\sigma_{y}$ is monotonic and smooth, the right-hand side of $(4.3)$ lies in $\mathscr{D}(0, \infty)$ whenever $\psi$ does, and we see that

$$
\begin{aligned}
\|\Omega(z) \psi\|^{2} & =\frac{1}{y^{2}} \int_{0}^{\infty} r \gamma_{y}^{\prime}(r)^{2}\left|\psi\left(\sigma_{y}(r)\right)\right|^{2} d r \\
& =\frac{1}{y^{2}} \int_{0}^{\infty} \sigma_{y}(s) \gamma_{y}^{\prime}\left(\sigma_{y}(s)\right)^{2}|\psi(s)|^{2}\left|\sigma_{y}^{\prime}(s)\right| d s \\
& =\frac{1}{y^{2}} \int_{0}^{\infty} s \sigma_{y}(s) \gamma_{y}^{\prime}(s) \gamma_{y}^{\prime}\left(\sigma_{y}(s)\right)|\psi(s)|^{2} \frac{d s}{s},
\end{aligned}
$$

where (3.5) has been used. Since $s \mapsto s \gamma_{y}^{\prime}(s)$ increases from 1 to $+\infty$ for $0<s<\infty$, no bound of the form $\|\Omega(i) \psi\| \leq C\|\psi\|$ is possible. We remark that the equivalent operators are (of course) bounded for compact groups, and for the Heisenberg group. But unboundedness is not unheard of, as it happens for the Poincaré group [19], Section 4.

The $\Omega(z)$ are hermitian on the domain $\mathscr{D}(0, \infty)$. For $\phi, \psi$ in this domain, we get

$$
\begin{aligned}
\langle\phi \mid \Omega(i) \psi\rangle & =\int_{0}^{\infty} \overline{\phi(r)} \Omega(i) \psi(r) \frac{d r}{r}=\int_{0}^{\infty} \overline{\phi(r)} \gamma^{\prime}(r) \psi(\sigma(r)) d r \\
& =\int_{0}^{\infty} \overline{\phi(\sigma(s))} \gamma^{\prime}(\sigma(s)) \psi(s)\left|\sigma^{\prime}(s)\right| d s \\
& =\int_{0}^{\infty} s \gamma^{\prime}(s) \overline{\phi(\sigma(s))} \psi(s) \frac{d s}{s}=\langle\Omega(i) \phi \mid \psi\rangle .
\end{aligned}
$$

Likewise, $\langle\phi \mid \Omega(z) \psi\rangle=\langle\Omega(z) \phi \mid \psi\rangle$ by covariance, since $U^{\dagger}\left(g_{z}\right)$ preserves $\mathscr{D}(0, \infty)$. We prove that $\Omega(z)$ is closable and identify its closure, and then show selfadjointness. 
This is easy to do using the fortunate fact that its square is a multiplication operator, $\Omega^{2}(z)=M_{\eta_{z}^{2}}$, where the unbounded positive function $\eta_{z}$ is given by

$$
\eta_{z}^{2}(r):=r y^{-2} \sigma_{y}(r) \gamma_{y}^{\prime}(r) \gamma_{y}^{\prime}\left(\sigma_{y}(r)\right)
$$

Notice that $\eta_{z}(r)=\eta_{i}(r / y)$ for $z \in \Pi$. The natural domains for the several $\Omega(z)$ are the dense subspaces $B_{z}$ of $\mathcal{K}$ defined as

$$
B_{z}:=\left\{\psi \in \mathcal{K}: \eta_{z} \psi \in \mathcal{K}\right\} .
$$

Note that (4.6) remains valid for $\phi, \psi \in B_{z}$ so that $\Omega(z)$ is hermitian on this domain.

Proposition 4.1. On the respective domains $B_{z}$, the operators $\Omega(z)$ are selfadjoint.

Proof. First, take $\psi \in \mathscr{D}(0, \infty)$. One sees at once that indeed $\Omega^{2}(z) \psi(r)=$ $\eta_{z}^{2}(r) \psi(r)$. We already showed that $\|\Omega(z) \psi\|=\left\|\eta_{z} \psi\right\|$, using (4.3); and this continues to hold for all $\psi \in B_{z}$. Observe that $B_{z}$ is complete in the graph norm given by $\|\psi \mid\|^{2}:=\|\psi\|^{2}+\left\|\eta_{z} \psi\right\|^{2}$, and that $\mathscr{D}(0, \infty)$ is dense in $B_{z}$ for this norm. Thus $\Omega(z)$, with domain $B_{z}$, is a closed operator. Clearly $\operatorname{Dom} \Omega(z) \subset \operatorname{Dom} \Omega(z)^{\dagger}$ since $\Omega(z)$ is hermitian on $B_{z}$.

Note that if $\psi \in \operatorname{Dom} \Omega^{\dagger}(z)$, and if $\chi_{n}$ is the indicator function of the interval $[1 / n, n]$, say, then $\chi_{n} \Omega(z)^{\dagger} \psi \in B_{z}$ and a routine argument, using the monotone convergence theorem, shows that

$$
\left\|\Omega(z)^{\dagger} \psi\right\|=\lim _{n \rightarrow \infty}\left\|\chi_{n} \Omega(z)^{\dagger} \psi\right\|=\lim _{n \rightarrow \infty} \sup _{\|\phi\|=1}\left|\left\langle\Omega(z)\left(\chi_{n} \phi\right) \mid \psi\right\rangle\right|=\cdots=\left\|\eta_{z} \psi\right\|,
$$

so that necessarily $\psi \in B_{z}$. Thus $\operatorname{Dom} \Omega(z)^{\dagger}=B_{z}=\operatorname{Dom} \Omega(z)$, as required.

Note also that the original domain $\mathscr{D}(0, \infty)$, being dense in each $B_{z}$ for the graph norm, is a common core for all the $\Omega(z)$, which are therefore essentially selfadjoint on that domain.

A consequence of the operators $\Omega(z)$ being selfadjoint (not just formally so) is that they become observables in the quantum-mechanical sense. For the StratonovichWeyl operators in the standard (Heisenberg covariant) case, see the discussion in [71].

\subsection{Traciality}

Lemma 4.2. The quantizer is tracial, in the sense that

$$
\operatorname{Tr}(\Omega(w) \Omega(z))=y \delta(w-z) \text { for all } w, z \in \Pi,
$$

where the right-hand side is the reproducing kernel for the Hilbert space $L^{2}(\Pi, d x d y / y)$. 
Proof. With $w=u+i v, z=x+i y$, we obtain

$$
\begin{aligned}
\operatorname{Tr} & \Omega(w) \Omega(z)) \\
& =\int_{0}^{\infty} \int_{0}^{\infty} \Omega(w ; r, s) \Omega(z ; s, r) \frac{d r d s}{r s} \\
& =\int_{0}^{\infty} \int_{0}^{\infty} \frac{r s}{v y} \gamma^{\prime}\left(\frac{s}{v}\right) \gamma^{\prime}\left(\frac{r}{y}\right) e^{-2 \pi i(r-s)(x / y-u / v)} \delta\left(r-\sigma_{v}(s)\right) \delta\left(s-\sigma_{y}(r)\right) d r d s \\
& =\int_{0}^{\infty} \frac{r}{v} \sigma\left(\frac{r}{y}\right) \gamma^{\prime}\left(\frac{\sigma_{y}(r)}{v}\right) \gamma^{\prime}\left(\frac{r}{y}\right) e^{-2 \pi i(x / y-u / v)\left(r-\sigma_{y}(r)\right)} \delta\left(\sigma_{v}\left(\sigma_{y}(r)\right)-r\right) d r .
\end{aligned}
$$

The argument of this last delta vanishes if and only if $\sigma_{v}(r)=\sigma_{y}(r)$, that is, if and only if $v=y$. On setting $f(v):=\sigma_{v}\left(\sigma_{y}(r)\right)-r$, it follows that

$$
\begin{aligned}
\left.f^{\prime}(v)\right|_{v=y} & =\sigma\left(\sigma\left(\frac{r}{y}\right)\right)-\sigma^{\prime}\left(\sigma\left(\frac{r}{y}\right)\right) \sigma\left(\frac{r}{y}\right) \\
& =\frac{r}{y}-\sigma^{\prime}\left(\sigma\left(\frac{r}{y}\right)\right) \sigma\left(\frac{r}{y}\right)=\frac{r}{y} \sigma\left(\frac{r}{y}\right) \gamma^{\prime}\left(\sigma\left(\frac{r}{y}\right)\right),
\end{aligned}
$$

using (3.4). Therefore,

$$
\frac{r}{y} \sigma\left(\frac{r}{y}\right) \gamma^{\prime}\left(\sigma\left(\frac{r}{y}\right)\right) \delta\left(r-\sigma_{v}\left(\sigma_{y}(r)\right)\right)=\delta(v-y)
$$

and (4.5) follows at once:

$$
\operatorname{Tr}(\Omega(w) \Omega(z))=\delta(v-y) \int_{0}^{\infty} \gamma^{\prime}\left(\frac{r}{y}\right) e^{-2 \pi i(x-u) \gamma(r / y)} d r=y \delta(x-u) \delta(v-y) .
$$

Corollary 4.3. The maps (4.2) establish an isometric isomorphism between the Hilbert space of Hilbert-Schmidt operators on $\mathcal{K}$ and the Hilbert space $L^{2}(\Pi, d \omega(z))$ of square-summable functions on the upper Poincaré half-plane with the left-invariant measure.

4.5. Relation with the Wigner functions on the half-plane. Starting from (4.4a), by dequantization we obtain

$$
\begin{aligned}
W_{A}(z) & =\operatorname{Tr}(\Omega(z) A)=\int_{0}^{\infty} \int_{0}^{\infty} A(r, s) \Omega(z ; s, r) \frac{d r}{r} \frac{d s}{s} \\
& =\frac{1}{y} \int_{0}^{\infty} \int_{0}^{\infty} A(r, s) \gamma^{\prime}(s / y) e^{2 \pi i x \gamma(s / y)} \delta\left(r-\sigma_{y}(s)\right) d r d s \\
& =\frac{1}{y} \int_{0}^{\infty} A\left(\sigma_{y}(s), s\right) \gamma^{\prime}(s / y) e^{2 \pi i x \gamma(s / y)} d s \\
& =\int_{-\infty}^{\infty} A(y \lambda(u), y \lambda(-u)) e^{-2 \pi i x u} d u .
\end{aligned}
$$


Note how by means of (4.4b) we recover the kernel of $A$ from its dequantization:

$$
A(r, s)=\int_{\Pi} W_{A}(z) \Omega(z ; r, s) \frac{d x d y}{y}=\int_{-\infty}^{\infty} W_{A}\left(x, \frac{r-s}{\log (r / s)}\right) e^{2 \pi i x \log (r / s)} d x .
$$

In particular, the Wigner function $W^{\psi}$ of a state $\psi$ is simply the expected value of the Stratonovich-Weyl operator $\Omega$ or the dequantization of the projector $|\psi\rangle\langle\psi|$ :

$$
W^{\psi}(z):=\langle\psi \mid \Omega(z) \psi\rangle=\langle\Omega(z) \psi \mid \psi\rangle=\operatorname{Tr}(\Omega(z)|\psi\rangle\langle\psi|) .
$$

The operator $|\psi\rangle\langle\psi|$ has kernel $A(r, s)=\psi(r) \psi^{*}(s)$. Thus,

$$
W^{\psi}(z)=\int_{\mathbb{R}} e^{-2 \pi i x u} \psi(y \lambda(u)) \psi^{*}(y \lambda(-u)) d u .
$$

Now, for the upper half-plane, a plethora of "affine Wigner functions" were originally constructed by the Bertrands [10]; see also [59]. One family among several options satisfying good covariance properties (under the ' $a x+b$ ' group and some extensions of it) is distinguished by "unitarity", that is, the correspondence $|\psi\rangle\langle\psi| \rightarrow W^{\psi}$ should extend to a unitary isomorphism between Hilbert-Schmidt operators and $L^{2}(\Pi, d x d y / y)$. For our purposes, it is enough to check that (4.9) with our quantizer coincides with their Wigner functions. This is done by inspection of formula (57) in [59], modulo our conventions for the unirrep $K\left[\mathcal{O}_{+}\right]$, or equation (IV.7) in [11], where Darboux coordinates on the phase space are used.

For an arbitrary normalized state $\psi$, we remark that $\int_{\Pi}\left|W^{\psi}(z)\right|^{2} d \omega(z)=1$, which seems curious since $\psi$ need not belong to the domains of all $\Omega(z)$. We return to this question in [41]. Geometrical properties of the affine Wigner functions have been much investigated, in regard to positivity, localization, marginal distributions, interference, etc. On this, we can do little better than to refer to Flandrin's articles [33], [34].

4.6. Summary. The strategy outlined in Section 4.1 has been successful. The outcome is that the deceptively simple formula for the operator-valued distribution

$$
\mathscr{D}^{\prime}(\Pi) \hat{\otimes} \mathcal{B}(\mathcal{K}) \ni \Omega(F):=\int_{\text {aff }} e^{-2 \pi i\langle F \mid X\rangle} U(\exp X) d X,
$$

with $d X$ the Lebesgue measure, makes sense, and the bounded operators

$$
\int_{\text {aff }} a(F) \int_{\text {aff }} e^{-2 \pi i\langle F \mid X\rangle} U(\exp X) d X d F
$$

for $a$ a test function on $a_{f f}{ }^{*}$, supported on $\Pi$, are explicitly given. Lest the reader be misled by the heuristic approach ostensibly taken in Section 4.1 , it must be said that (4.10) recommends itself because from it covariance of $\Omega$ is ensured to hold by an abstract argument. Again, the reader will have no difficulty in checking it. This point of view had been emphasized in [75]. 


\section{The Moyal twisted product on the half-plane}

The Moyal product $f \star h$ of two functions $f, h$ on $\Pi$ is by definition the dequantization of the operator $Q(f) Q(h)$; to wit,

$$
\begin{aligned}
f \star h(z): & =\operatorname{Tr}\left[\Omega(z) \int f(w) \Omega(w) d \omega(w) \int g(t) \Omega(t) d \omega(t)\right] \\
& =\iint_{\Pi^{2}} K_{\star}(z, w, t) f(w) h(t) d \omega(w) d \omega(t),
\end{aligned}
$$

where

$$
K_{\star}(z, w, t):=\operatorname{Tr}(\Omega(z) \Omega(w) \Omega(t)) .
$$

The "trikernel" $K_{\star}$ enjoys left invariance:

$$
\begin{aligned}
\operatorname{Tr}(\Omega(Z \cdot z) \Omega(Z \cdot w) \Omega(Z \cdot t)) & =\operatorname{Tr}\left(U\left(g_{Z}\right) \Omega(z) \Omega(w) \Omega(t) U^{\dagger}\left(g_{Z}\right)\right) \\
& =\operatorname{Tr}(\Omega(z) \Omega(w) \Omega(t)) .
\end{aligned}
$$

Using this invariance we can rewrite the Moyal product of two functions - also, using (1.2) and the cyclicity of the trace-integral, eventually of many distributions [44] on $\Pi$, gifted with the invariant measure $d \omega(z)=d x d y / y$, in the following ways:

$$
\begin{aligned}
f \star h(z) & =\iint_{\Pi^{2}} K_{\star}(z, w, t) f(w) h(t) d \omega(w) d \omega(t) \\
& =\iint_{\Pi^{2}} K_{\star}\left(i, z^{-1} \cdot w, z^{-1} \cdot t\right) f(w) h(t) d \omega(w) d \omega(t) \\
& =\iint_{\Pi^{2}} K_{\star}(i, w, t) f(z \cdot w) h(z \cdot t) d \omega(w) d \omega(t) .
\end{aligned}
$$

Let $R_{w}, R_{t}$ denote right multiplication operators (i.e., the right regular action) for the group structure on $\Pi$, and note the elegance of the final "deformation" formula:

$$
f \star h=\iint_{\Pi^{2}} K_{\star}(i, w, t) R_{w} f R_{t} h d \omega(w) d \omega(t) .
$$

We do not omit, finally, the tracial identity for our star product:

$$
\int_{\Pi} f \star h(z) d \omega(z)=\int_{\Pi} f(z) h(z) d \omega(z) ;
$$

this comes straight from (5.1) on using the properties of the Stratonovich-Weyl quantizer. 
5.1. The trikernel for the twisted product and its symmetries. We need the solution $\kappa\left(y_{0}, y_{1}, y_{2}\right)$ of the equation

$$
s=\sigma_{y_{0}}\left(\sigma_{y_{1}}\left(\sigma_{y_{2}}(s)\right)\right) .
$$

Equivalently, it is the solution of

$$
\sigma_{y_{0}}(s)=\sigma_{y_{1}}\left(\sigma_{y_{2}}(s)\right) \quad \text { or } \quad \sigma_{y_{2}}(s)=\sigma_{y_{1}}\left(\sigma_{y_{0}}(s)\right) .
$$

Given any (positive) values of $y_{0}, y_{1}, y_{2}$, there is indeed a unique solution to these equations, for one of the sides increases monotonically from 0 to $\infty$, whereas the other decreases from $\infty$ to 0 . Under the exchange $z_{0} \leftrightarrow z_{2}$, with $z_{1}$ held fixed, the value of $\kappa$ is unchanged. Moreover, $\kappa$ is a homogeneous function of degree 1 , since $c^{-1} \kappa\left(c y_{0}, c y_{1}, c y_{2}\right)$ and $\kappa\left(y_{0}, y_{1}, y_{2}\right)$ solve the same equation. We abbreviate $\sigma_{012}:=\sigma_{y_{0}} \circ \sigma_{y_{1}} \circ \sigma_{y_{2}}$, whose inverse function is $\sigma_{210}:=\sigma_{y_{2}} \circ \sigma_{y_{1}} \circ \sigma_{y_{0}}$.

With this in hand, one can proceed to compute the trikernel. After a straightforward, though tedious, calculation, one obtains

$$
\begin{gathered}
K_{\star}\left(z_{0}, z_{1}, z_{2}\right)=\frac{\kappa \sigma_{y_{0}}(\kappa) \sigma_{y_{2}}(\kappa) \gamma_{y_{2}}^{\prime}(\kappa) \gamma_{y_{0}}^{\prime}\left(\sigma_{y_{0}}(\kappa)\right) \gamma_{y_{1}}^{\prime}\left(\sigma_{y_{2}}(\kappa)\right)}{y_{0} y_{1} y_{2}\left(1-\sigma_{012}^{\prime}(\kappa)\right)} \\
\cdot e^{2 \pi i\left[\frac{x_{0}}{y_{0}} \gamma_{y_{0}}(\kappa)-\frac{x_{1}}{y_{1}} \gamma_{y_{1}}\left(\sigma_{y_{2}}(\kappa)\right)-\frac{x_{2}}{y_{2}} \gamma_{y_{2}}(\kappa)\right],}
\end{gathered}
$$

where the dependence on $y_{0}, y_{1}, y_{2}$ through $\kappa$ is understood.

The trikernel is of the general form

$$
K_{\star}\left(z_{0}, z_{1}, z_{2}\right)=A\left(z_{0}, z_{1}, z_{2}\right) e^{2 \pi i S\left(z_{0}, z_{1}, z_{2}\right)}
$$

for real amplitude $A: M^{3} \rightarrow \mathbb{R}$ and phase $S: M^{3} \rightarrow \mathbb{R}$ functions. By its construction, we expect $K_{\star}$ to have several symmetries. First of all, cyclical symmetry. With $\hat{\kappa}\left(y_{0}, y_{1}, y_{2}\right)$ defined by $\hat{\kappa}=\sigma_{201}(\hat{\kappa})$, with the obvious notation, the same calculation gives

$$
\begin{gathered}
K_{\star}\left(z_{2}, z_{0}, z_{1}\right)=\frac{\hat{\kappa} \sigma_{y_{2}}(\hat{\kappa}) \sigma_{y_{1}}(\hat{\kappa}) \gamma_{y_{1}}^{\prime}(\hat{\kappa}) \gamma_{y_{2}}^{\prime}\left(\sigma_{y_{2}}(\hat{\kappa})\right) \gamma_{y_{0}}^{\prime}\left(\sigma_{y_{1}}(\hat{\kappa})\right)}{y_{0} y_{1} y_{2}\left(1-\sigma_{201}^{\prime}(\hat{\kappa})\right)} \\
\cdot e^{2 \pi i\left[\frac{x_{2}}{y_{2}} \gamma_{y_{2}}(\hat{\kappa})-\frac{x_{0}}{y_{0}} \gamma_{y_{0}}\left(\sigma_{y_{1}}(\hat{\kappa})\right)-\frac{x_{1}}{y_{1}} \gamma_{y_{1}}(\hat{\kappa})\right]} .
\end{gathered}
$$

But $\hat{\kappa}$ is just $\sigma_{y_{2}}(\kappa)$; thus

$$
\gamma_{y_{2}}(\kappa)=-\gamma_{y_{2}}(\hat{\kappa}) \text { and also } \sigma_{y_{0}}(\kappa)=\sigma_{y_{1}}(\hat{\kappa}) \text {, implying } \gamma_{y_{0}}\left(\sigma_{y_{1}}(\hat{\kappa})\right)=-\gamma_{y_{0}}(\kappa) \text {, }
$$

and one sees at once that the numerator of the fraction in (5.4) and the phase factor coincide with those of the new formula (5.5). Moreover,

$$
\begin{aligned}
\sigma_{201}^{\prime}(\hat{\kappa}) & =\sigma_{y_{2}}^{\prime}\left(\sigma_{y_{0}}\left(\sigma_{y_{1}}(\hat{\kappa})\right)\right) \sigma_{y_{0}}^{\prime}\left(\sigma_{y_{1}}(\hat{\kappa})\right) \sigma_{y_{1}}^{\prime}(\hat{\kappa}) \\
& =\sigma_{y_{2}}^{\prime}(\kappa) \sigma_{y_{0}}^{\prime}\left(\sigma_{y_{1}}\left(\sigma_{y_{2}}(\kappa)\right)\right) \sigma_{y_{1}}^{\prime}\left(\sigma_{y_{2}}(\kappa)\right)=\sigma_{012}^{\prime}(\kappa),
\end{aligned}
$$


and we conclude that, as expected,

$$
K_{\star}\left(z_{0}, z_{1}, z_{2}\right)=K_{\star}\left(z_{2}, z_{0}, z_{1}\right)=K_{\star}\left(z_{1}, z_{2}, z_{0}\right) .
$$

Next we investigate the switch $z_{0} \leftrightarrow z_{2}$ (with $z_{1}$ fixed). We observe that

$$
\frac{1}{1-\sigma_{012}^{\prime}(\kappa)}=\frac{-\sigma_{210}^{\prime}\left(\sigma_{210}(\kappa)\right)}{1-\sigma_{210}^{\prime}\left(\sigma_{210}(\kappa)\right)}=\frac{-\sigma_{210}^{\prime}(\kappa)}{1-\sigma_{210}^{\prime}(\kappa)},
$$

since $\sigma_{210}(\kappa)=\kappa$. On multiplying the numerator of the fraction occurring in (5.4) by

$$
-\sigma_{210}^{\prime}(\kappa)=-\sigma_{y_{2}}^{\prime}\left(\sigma_{y_{1}}\left(\sigma_{y_{0}}(\kappa)\right)\right) \sigma_{y_{1}}^{\prime}\left(\sigma_{y_{0}}(\kappa)\right) \sigma_{y_{0}}^{\prime}(\kappa)
$$

and taking (3.5) into account, the whole fraction becomes

$$
\frac{\kappa \sigma_{y_{0}}(\kappa) \sigma_{y_{2}}(\kappa) \gamma_{y_{2}}^{\prime}\left(\left(\sigma_{y_{2}}(\kappa)\right) \gamma_{y_{1}}^{\prime}\left(\sigma_{y_{0}}(\kappa)\right) \gamma_{y_{0}}^{\prime}(\kappa)\right.}{y_{0} y_{1} y_{2}\left(1-\sigma_{210}^{\prime}(\kappa)\right)} .
$$

In other words, the fraction is unchanged by the switch $z_{0} \leftrightarrow z_{2}$. Now note that

$$
\gamma_{y_{1}}\left(\sigma_{y_{2}}(\kappa)\right)=\sigma_{y_{2}}(\kappa)-\sigma_{y_{1}}\left(\sigma_{y_{2}}(\kappa)\right)=\sigma_{y_{2}}(\kappa)-\sigma_{y_{0}}(\kappa)=\gamma_{y_{0}}(\kappa)-\gamma_{y_{2}}(\kappa) .
$$

Using this formula, we can reexpress the phase factor in the trikernel (5.4) as

$$
\exp \left\{2 \pi i\left[\left(\frac{x_{0}}{y_{0}}-\frac{x_{1}}{y_{1}}\right) \gamma_{y_{0}}(\kappa)+\left(\frac{x_{1}}{y_{1}}-\frac{x_{2}}{y_{2}}\right) \gamma_{y_{2}}(\kappa)\right]\right\} \text {. }
$$

This is manifestly skewsymmetric under the exchange $z_{0} \leftrightarrow z_{2}$, with $z_{1}$ held fixed. In fine, we have shown that

$$
\overline{K_{\star}\left(z_{0}, z_{1}, z_{2}\right)}=K_{\star}\left(z_{2}, z_{1}, z_{0}\right)=K_{\star}\left(z_{0}, z_{2}, z_{1}\right)=K_{\star}\left(z_{1}, z_{0}, z_{2}\right),
$$

where cyclic symmetry has been reinvoked; in particular, this confirms that complex conjugation is an antilinear involution for the twisted product. Corollary 4.3 can now be read as stating that $\left(L^{2}(\Pi, d \omega), \star\right)$ is a Hilbert algebra.

Finally, we expect $K_{\star}\left(z_{0}, z_{1}, z_{2}\right)=K_{\star}\left(i, z_{0}^{-1} \cdot z_{1}, z_{0}^{-1} \cdot z_{2}\right)$, in view of left invariance. Indeed, the trikernel is invariant under the transformations

$$
\begin{array}{llll}
x_{0} \mapsto 0, & x_{1} \mapsto x_{1}-x_{0} y_{1} / y_{0}, & x_{2} \mapsto x_{2}-x_{0} y_{2} / y_{0}, \\
y_{0} \mapsto 1, & y_{1} \mapsto y_{1} / y_{0}, & y_{2} \mapsto y_{2} / y_{0},
\end{array}
$$

on account of the homogeneity properties of $\kappa$.

Inspired by earlier exact results [42], [82], Weinstein [81] developed a heuristic argument for the construction of trikernels on symplectic symmetric spaces. In this approach, the phase function $S$ is postulated to be an (invariant) oriented symplectic area of a geodesic triangle for which $z_{0}, z_{1}, z_{2}$ are the midpoints of the sides, and the 
amplitude $A$ is chosen as to achieve associativity of the twisted product - implicit in our treatment - and other desirable properties. The idea has been further developed in [69] - where some caveats are made - and in [12] and [24, § 3.3.5]. It is sometimes linked to the purported role of reflections in producing quantizers. However, it is known [76] that reflections do not lead directly to Stratonovich-Weyl quantizers in general; and it is straightforward to verify that, although it enjoys the same symmetries, our phase function is not the area. The question deserves further investigation [13].

5.2. The extended covariance group of the twisted product. The ordinary Moyal product on the full plane $\mathbb{R}^{2}$ has a larger covariance group than the original Heisenberg group of phase-space translations under which it is equivariant; this is the inhomogeneous metaplectic group of unitaries $U(g)$ such that $Q(f) \mapsto U(g) Q(f) U^{\dagger}(g)=$ : $Q(f \circ \varphi)$ implements a diffeomorphism $\varphi$ of the plane that normalizes the action of the Heisenberg group. For the half-plane $\Pi$, its analogue will be a Lie group of symplectomorphisms normalizing the action of Aff. At the infinitesimal level, the generators of this group are given by symbols $f_{i}$ such that the Moyal bracket

$$
\left[f_{i}, h\right]_{\star}:=2 \pi i\left(f_{i} \star h-h \star f_{i}\right)
$$

coincides, for arbitrary $h$, with the Poisson bracket

$$
\left\{f_{i}, h\right\}_{\mathrm{PB}}=y\left(\frac{\partial f_{i}}{\partial x} \frac{\partial h}{\partial y}-\frac{\partial f_{i}}{\partial y} \frac{\partial h}{\partial x}\right)
$$

corresponding to the symplectic 2-form $d x \wedge d y / y$ on $\Pi$. In other words, these $f_{i}$ are "distinguished observables" in the sense of [9].

The (neutral component of) the normalizer of Aff within the group of symplectomorphisms of $\Pi$ is easily determined [75]. Any one-parameter subgroup is generated by a Hamiltonian vector field of the form $H_{f}=y\left(f_{y} \frac{\partial}{\partial x}-f_{x} \frac{\partial}{\partial y}\right)$ for some $f \in C^{\infty}(\Pi)$. Since the action of aff on $\Pi$ is generated by the vector fields $y \frac{\partial}{\partial x}$ and $y \frac{\partial}{\partial y}$, we require that

$$
\begin{aligned}
& {\left[H_{f}, y \frac{\partial}{\partial x}\right]=-\left(f_{x y}+f_{y}\right) y \frac{\partial}{\partial x}+y^{2} f_{x x} \frac{\partial}{\partial y},} \\
& {\left[H_{f}, y \frac{\partial}{\partial y}\right]=-\left(y f_{y y}+f_{y}\right) y \frac{\partial}{\partial x}+y^{2} f_{x y} \frac{\partial}{\partial y}}
\end{aligned}
$$

be linear combinations of $y \frac{\partial}{\partial x}$ and $y \frac{\partial}{\partial y}$. This easily entails that

$$
f(x, y)=\alpha x+\beta y+\gamma \log y+\delta
$$

for some constants $\alpha, \beta, \gamma, \delta$. Ignoring the trivial constant term that does not contribute to $H_{f}=-\alpha y \frac{\partial}{\partial y}+\beta y \frac{\partial}{\partial x}+\gamma \frac{\partial}{\partial x}$, we obtain a solvable 3-parameter group $G$ extending 
Aff by $\mathbb{R}$. The appearance of the log function above is related to the existence of ray unirreps of $G$ given by

$$
U(a, b, c) \psi(r)=e^{2 \pi i b r} r^{2 \pi i c} \psi(a r) .
$$

This covariance group was also found in [10] by a not very different method.

To ascertain that this group is indeed a symmetry group of our twisted product, one must verify that the three functions $x, y, \log y$ are distinguished observables.

Lemma 5.1. For any smooth function $h$ on $\Pi$, the following relations hold:

$$
[x, h]_{\star}=y \frac{\partial h}{\partial y} ; \quad[y, h]_{\star}=-y \frac{\partial h}{\partial x} ; \quad[\log y, h]_{\star}=-\frac{\partial h}{\partial x} .
$$

Proof. We first determine the operator kernels corresponding, via (4.8), to the three basic functions:

$$
\begin{aligned}
Q_{x}(r, s) & =\int_{\mathbb{R}} x e^{2 \pi i x \log (r / s)} d x \\
& =\frac{1}{2 \pi i} \delta^{\prime}(\log r-\log s)=\frac{1}{2 \pi i}\left(s^{2} \delta^{\prime}(r-s)-s \delta(r-s)\right), \\
Q_{y}(r, s) & =\int_{\mathbb{R}} \frac{r-s}{\log (r / s)} e^{2 \pi i x \log (r / s)} d x \\
& =\frac{r}{\lambda(\log (r / s))} \delta(\log r-\log s)=\frac{r s}{\lambda(\log (r / s))} \delta(r-s), \\
Q_{\log y(r, s)} & =\int_{\mathbb{R}} \log \left(\frac{r-s}{\log (r / s)}\right) e^{2 \pi i x \log (r / s)} d x \\
& =\frac{r}{\lambda(\log (r / s))} \delta(\log r-\log s)=(s \log r-s \log \lambda(\log (r / s))) \delta(r-s)
\end{aligned}
$$

We have written $Q_{x}$ for $Q(x)$, and similarly for the other operators. If the quantized operator $Q(h)$ has kernel $B(s, t)$, then $Q\left([x, h]_{\star}\right)=2 \pi i\left[Q_{x}, Q(h)\right]$ has kernel

$$
\begin{aligned}
2 \pi i & \int_{0}^{\infty}\left(Q_{x}(r, t) B(t, s)-B(r, t) Q_{x}(t, s)\right) \frac{d t}{t} \\
& =\int_{0}^{\infty}\left(t \delta^{\prime}(r-t)-\delta(r-t)\right) B(t, s)-B(r, t)\left(t \delta^{\prime}(t-s)-\delta(t-s)\right) d t \\
& =\left.\frac{\partial}{\partial t}\right|_{t=r}(-t B(t, s))-\left.\frac{\partial}{\partial t}\right|_{t=s}(-t B(r, t))=-r \frac{\partial B}{\partial r}(r, s)+s \frac{\partial B}{\partial s}(r, s) .
\end{aligned}
$$


On the other hand, (4.7) yields

$$
\begin{aligned}
y \frac{\partial h}{\partial y}(z)= & \int_{\mathbb{R}}\left(y \lambda(u) \frac{\partial B}{\partial r}(y \lambda(u), y \lambda(-u))\right. \\
& \left.\quad+y \lambda(-u) \frac{\partial B}{\partial s}(y \lambda(u), y \lambda(-u))\right) e^{-2 \pi i x u} d u \\
= & \frac{1}{y} \int_{0}^{\infty} r \frac{\partial B}{\partial r}\left(r, \sigma_{y}(r)\right) \gamma_{y}^{\prime}(r) e^{-2 \pi i x \gamma(r / y)} d r \\
& \quad+\frac{1}{y} \int_{0}^{\infty} s \frac{\partial B}{\partial s}\left(\sigma_{y}(s), s\right) \gamma_{y}^{\prime}(s) e^{2 \pi i x \gamma(s / y)} d s \\
= & \frac{1}{y} \int_{0}^{\infty} \int_{0}^{\infty}\left(-r \frac{\partial B}{\partial r}+s \frac{\partial B}{\partial s}\right)(r, s) \gamma_{y}^{\prime}(s) e^{2 \pi i x \gamma(s / y)} \delta\left(r-\sigma_{y}(s)\right) d r d s,
\end{aligned}
$$

and using (4.7) once more we obtain the desired relation:

$$
\{x, h\}_{\mathrm{PB}}=y \frac{\partial h}{\partial y}=W_{Q\left([x, h]_{\star}\right)}=[x, h]_{\star} .
$$

The other cases are simpler. One finds from (5.7) that the kernel of $Q\left([y, h]_{\star}\right)=$ $2 \pi i\left[Q_{y}, Q(h)\right]$ is $2 \pi i(r-s) B(r, s)$ and that $Q\left([\log y, h]_{\star}\right)$ has kernel $2 \pi i(\log r-$ $\log s) B(r, s)$. Therefore,

$$
\begin{aligned}
{[y, h]_{\star}(z) } & =\frac{2 \pi i}{y} \int_{0}^{\infty}\left(\sigma_{y}(s)-s\right) B\left(\sigma_{y}(s), s\right) \gamma_{y}^{\prime}(s) e^{2 \pi i x \gamma(s / y)} d s \\
& =-2 \pi i \int_{0}^{\infty} B\left(\sigma_{y}(s), s\right) \gamma_{y}^{\prime}(s)\left(\gamma(s / y) e^{2 \pi i x \gamma(s / y)}\right) d s \\
& =-\frac{\partial}{\partial x} \int_{0}^{\infty} B\left(\sigma_{y}(s), s\right) \gamma_{y}^{\prime}(s) e^{2 \pi i x \gamma(s / y)} d s \\
& =-\frac{\partial}{\partial x}(y h(z))=-y \frac{\partial h}{\partial x}(z) .
\end{aligned}
$$

An almost identical calculation, with the relation $\sigma_{y}(s)-s=-y \gamma(s / y)$ replaced by the identity $\log \sigma_{y}(s)-\log s=-\gamma(s / y)$, yields $[\log y, h]_{\star}=-\partial h / \partial x$. Or we may just remark that $[\cdot, h]_{\star}$ is a derivation.

On regarding the functions $x, y$ on phase space as elements of the Lie algebra aff, the first two equalities of (5.6) show that our twisted product is an aff-invariant $\star$-quantization in the sense of [9], [36].

5.3. On the universal enveloping algebra product. By duality and symmetrization, the universal enveloping algebra $U(g)$ of a Lie algebra $g$ can be realized by an algebra $\mathcal{P}\left(\mathrm{g}^{*}\right)$ of polynomial functions on $\mathrm{g}^{*}$. Thus it makes sense to compare the (restriction 
to $\Pi$ of) the product on $\mathcal{U}(\mathrm{aff})$ as transferred to $\mathcal{P}\left(\mathrm{g}^{*}\right)$ with the twisted product; in our case the correspondence gives $X_{1} \mapsto x \equiv x_{1} ; X_{2} \mapsto y \equiv x_{2}$. Let us denote by $*$ that transported product. Its expression is complicated in general, but it is well known that one obtains

$$
\begin{aligned}
x_{i} * h= & x_{i} h+\sum_{n=1}^{\infty} B_{n} \sum_{k_{1} \ldots k_{n}}\left\{\operatorname{ad}\left(x_{k_{1}}\right) \ldots \operatorname{ad}\left(x_{k_{n}}\right)\right\}_{\mathrm{sym}}\left(x_{i}\right) \frac{\partial^{n} h}{\partial x_{k_{1}} \ldots \partial x_{k_{n}}} ; \\
h * x_{i}= & x_{i} h+\frac{1}{2} \sum_{j}\left[x_{j}, x_{i}\right] \frac{\partial h}{\partial x_{j}} \\
& +\sum_{n=2}^{\infty} B_{n} \sum_{k_{1} \ldots k_{n}}\left\{\operatorname{ad}\left(x_{k_{1}}\right) \ldots \operatorname{ad}\left(x_{k_{n}}\right)\right\}_{\mathrm{sym}}\left(x_{i}\right) \frac{\partial^{n} h}{\partial x_{k_{1}} \ldots \partial x_{k_{n}}},
\end{aligned}
$$

where the $k_{j}$ take the values 1 or 2 in all possible forms and $\{\ldots\}_{\text {sym }}$ means total symmetrization of the operations inside the curly brackets. The rule makes sense because the Lie products are supposed known: here $\left[x_{1}, x_{2}\right]=x_{2}$. For instance, we see that, if $h$ depends only on the second variable, then the series terminates, and $x * h(x, y)=x h(y)+\frac{1}{2} y h^{\prime}(y)$. The second term of the series in (5.8) is just $\frac{1}{2}$ times the Poisson bracket:

$$
\sum_{j}\left[x_{j}, x\right] \frac{\partial h}{\partial x_{j}}=-y \frac{\partial h}{\partial y} ; \quad \sum_{j}\left[x_{j}, y\right] \frac{\partial h}{\partial x_{j}}=y \frac{\partial h}{\partial x} .
$$

Thus in particular

$$
x_{i} * h-h * x_{i}=\{x, h\}_{\mathrm{PB}}=\left[x_{i}, h\right]_{\star} .
$$

A detailed comparison between $*$ and the asymptotic expansion of $\star$ would lengthen this paper too much; we come back on this matter in [41] and [13].

\section{Right-covariant quantization}

In this section the notation $d_{l} z$ will be used for $d \omega(z)=d x d y / y$, and $d_{r} z$ for $d x d y / y^{2}$. For the purposes outlined in the introduction, we are actually interested in a right-covariant star product, as well as the left-covariant one constructed so far. We summarize again our desideratum: a pair of quantizers $\Omega^{L}, \Omega^{R}$, both acting on $\mathcal{K}$, satisfying

(i) $\Omega^{L, R}(z)^{\dagger}=\Omega^{L, R}(z)$,

(ii) $U\left(g_{z^{\prime}}\right) \Omega^{L}(z) U^{\dagger}\left(g_{z^{\prime}}\right)=\Omega^{L}\left(z^{\prime} \cdot z\right)$ and $U^{\dagger}\left(g_{z^{\prime}}\right) \Omega^{R}(z) U\left(g_{z^{\prime}}\right)=\Omega^{R}\left(z \cdot z^{\prime}\right)$,

(iii) $\operatorname{Tr} \Omega^{L, R}(z)=1$,

(iv) $\operatorname{Tr}\left(\Omega^{L, R}(z) \Omega^{L, R}\left(z^{\prime}\right)\right)=I_{L, R}\left(z, z^{\prime}\right)$, 
with $I_{L}$ and $I_{R}$ denoting the reproducing kernels for $L^{2}\left(\Pi, d_{l} z\right)$ and $L^{2}\left(\Pi, d_{r} z\right)$, respectively. Here $\Omega^{L} \equiv \Omega$ is the quantizer already found. Define

$$
\breve{f}(z):=f\left(z^{-1}\right) \quad \text { with } z^{-1}=(x+i y)^{-1}:=-x / y+i / y,
$$

the inverse for the product (2.7). It seems natural to replace the quantization rule (4.2) by

$$
A=\int_{\Pi} \Omega^{R}(z) f(z) d_{r} z=: Q_{R}(f),
$$

when using the right-covariant quantizer. We obtain $Q_{R}(\breve{f})=Q(f)$ if we declare that

$$
\Omega^{R}(z) \equiv \Omega\left(z^{-1}\right)
$$

It is not idle to check consistency of this rule with previous use of the coadjoint action and the diffeomorphism $z \mapsto g_{z}$. We need to verify that

$$
z \triangleleft g_{z^{\prime}} \equiv\left(g_{z^{\prime}}^{-1} \triangleright z^{-1}\right)^{-1}=z \cdot z^{\prime} .
$$

Indeed,

$$
\left(\left(y^{\prime},-x^{\prime}\right) \triangleright(-x / y+i / y)\right)^{-1}=\left(\frac{-x^{\prime}-x y^{\prime}}{y y^{\prime}}+\frac{i}{y y^{\prime}}\right)^{-1}=x^{\prime}+x y^{\prime}+i y y^{\prime} .
$$

The right-covariant quantizer is thus given by the following expression:

$$
\Omega^{R}(z)=\frac{1}{y} \iint_{\mathbb{R}^{2}} e^{2 \pi i(u x / y-v / y)} U\left(e^{u}, v / \lambda(-u)\right) d u d v .
$$

It is easy to check consistency of this with $\Omega(z)=\Omega^{R}\left(z^{-1}\right)$. It is also straightforward to verify, along the same lines as before, the four properties listed above; in particular,

$$
\operatorname{Tr}\left(\Omega^{R}(z) \Omega^{R}(w)\right)=y^{2} \delta(z-w) .
$$

For the trikernel, one obtains

$$
K_{\star}^{R}(z, w, t)=\operatorname{Tr}\left(\Omega^{R}(z) \Omega^{R}(w) \Omega^{R}(t)\right)=K_{\star}\left(z^{-1}, w^{-1}, t^{-1}\right) .
$$

This yields the following tautological relation between the twisted products $\star$ and $\star R$ :

$$
\begin{aligned}
& f \star^{R} h(z)=\int_{\Pi^{2}} K_{\star}^{R}(z, w, t) f(w) h(t) d_{r} w d_{r} t \\
& =\int_{\Pi^{2}} K_{\star}\left(z^{-1}, w^{-1}, t^{-1}\right) f(w) h(t) d_{r} w d_{r} t
\end{aligned}
$$

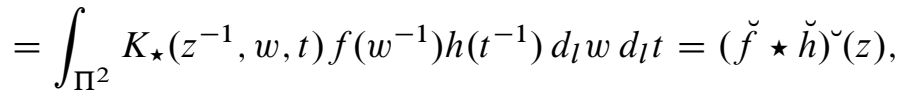


consistent with

$$
Q_{R}\left(f \star^{R} h\right)=Q_{R}(f) Q_{R}(h) .
$$

We finally register the following formula for the product $\star^{R}$, similar to (5.3):

$$
f \star^{R} h=\iint_{\Pi^{2}} K_{\star}(i, w, t) L_{w} f L_{t} h d_{l} w d_{l} t,
$$

and the tracial identity for the $\star^{R}$ product:

$$
\int_{\Pi} f \star^{R} h(z) d_{r} z=\int_{\Pi} f(z) h(z) d_{r} z .
$$

\section{Fourier-Moyal transformations on the ' $a x+b$ ' group}

7.1. The Fourier-Moyal kernels. Consider the following distribution or kernel:

$$
\mathbb{E}(z, g) \equiv \mathbb{E}_{L}(z, g):=\operatorname{Tr}\left(\Omega_{ \pm}(z) U_{ \pm}(g)\right)=W_{U_{ \pm}(g)}(z),
$$

for $z \in \pm \Pi$, respectively, and $g \in$ Aff. As a consequence of this definition, we see that $\mathbb{E}$ is the symbol for the unirreps:

$$
U_{ \pm}(g)=\int_{ \pm \Pi} \mathbb{E}(z, g) \Omega(z) d \omega_{ \pm}(z) .
$$

We compute the kernel, expecting the covariance property

$$
\mathbb{E}\left(h \triangleright z, h g h^{-1}\right)=\mathbb{E}(z, g) .
$$

It comes as a "nice surprise" that the kernel is a $U(1)$-valued smooth function. Take $\operatorname{Im} z>0$. From equation (4.1), one gets

$$
\Omega(z) U(g)=y \iint_{\mathbb{R}^{2}} e^{-2 \pi i(x u+y v)} U\left(e^{u} a, e^{u}(b+v / \lambda(u)) d u d v,\right.
$$

and the kernel of this operator is thus

$$
\begin{aligned}
\Omega U(z, g ; r, s) & =y \iint_{\mathbb{R}^{2}} e^{-2 \pi i(x u+y v)} e^{2 \pi i e^{u}(b+v / \lambda(u)) r} s \delta\left(s-r e^{u} a\right) d u d v \\
& =y \iint_{\mathbb{R}^{2}} e^{-2 \pi i(x u+y v)} e^{2 \pi i e^{u}(b+v / \lambda(u)) r} \delta(u-\log (s / r a)) d u d v \\
& =y \int_{\mathbb{R}} e^{-2 \pi i(x \log (s / r a)+y v)} e^{2 \pi i a^{-1} s(b+v / \lambda(\log (s / r a)))} d v \\
& =y e^{-2 \pi i x \log (s / r a)} e^{2 \pi i b s / a} \delta(y-s / a \lambda(\log (s / r a))) .
\end{aligned}
$$


Its trace is the desired kernel, explicitly:

$$
\begin{aligned}
\mathbb{E}(z, g) & =\int_{0}^{\infty} \Omega U(z, g ; r, r) \frac{d r}{r} \\
& =y e^{2 \pi i x \log a} \int_{0}^{\infty} e^{2 \pi i r b / a} \delta(y-r / \lambda(\log a)) \frac{d r}{r} \\
& =y e^{2 \pi i x \log a} \int_{0}^{\infty} e^{2 \pi i r b / a} \lambda(\log a) \delta(r-y \lambda(\log a)) \frac{d r}{r} \\
& =e^{2 \pi i(x \log a+y b \lambda(\log a) / a)}
\end{aligned}
$$

We then see that $\mathbb{E}$ is smooth and of modulus 1 . We check the coadjoint covariance of this kernel. With $h=\left(a^{\prime}, b^{\prime}\right)$, one indeed finds that

$$
\mathbb{E}\left(x+b^{\prime} y / a^{\prime}+i y / a^{\prime} ;\left(a, a^{\prime} b+b^{\prime}(1-a)\right)\right)=\mathbb{E}(x+i y ;(a, b)),
$$

since $\left(a^{-1}-1\right) \lambda(\log a)=-\log a$ from the definition (2.4). By computing on the second orbit, the formula is valid for $y<0$ as well.

Similarly, the right-covariant quantizer yields another kernel:

$$
\mathbb{E}_{R}(z, g):=\operatorname{Tr}\left(\Omega^{R}(z) U(g)\right)=\mathbb{E}\left(z^{-1}, g\right),
$$

with the expected covariance property:

$$
\mathbb{E}_{R}\left(z \triangleleft h, h^{-1} g h\right)=\mathbb{E}_{R}(z, g),
$$

which follows from (7.2), in view of (6.1). Thus we get, explicitly,

$$
\mathbb{E}_{R}(z, g)=e^{2 \pi i(-x \log a+b \lambda(\log a) / a) / y} .
$$

It is enlightening to pass to Lie-algebra coordinates in the $\mathbb{E}$-functions, for the first group arguments:

$$
\mathbb{E}(z ; u, b)=e^{2 \pi i(u x+b \lambda(-u) y)}, \quad \mathbb{E}_{R}(z ; u, b)=e^{2 \pi i(-u x+b \lambda(-u)) / y} ;
$$

or for both:

$$
\mathbb{E}(z ; u, v)=e^{2 \pi i(u x+v y)}, \quad \mathbb{E}_{R}(z ; u, v)=e^{2 \pi i(-u x / y+v / y)} .
$$

Note the simplicity of the last result for $\mathbb{E}$. If we regard $z$ as an element of $a f f^{*}$, then

$$
\operatorname{Tr}\left(\Omega_{ \pm}(z) U_{ \pm}(\exp X)\right)=\exp (2 \pi i\langle z \mid X\rangle) \text { for all } X \in \text { aff }
$$

has been proved to hold. 
In conclusion, the left Fourier-Moyal transformation, denoted $\mathbb{F}_{\mathrm{M}}$, is given by

$$
\begin{aligned}
\mathbb{F}_{\mathrm{M}}[f](z): & =\int \mathbb{E}(z, g) f(g) d_{l} g \\
& =\iint_{\mathrm{aff}} \exp \{2 \pi i(u x+v y)\} f\left(e^{u}, v / \lambda(-u)\right) \frac{d u d v}{\lambda(u)} \\
& =\iint_{\mathrm{Aff}} \exp \{2 \pi i(x \log a+b \lambda(\log a) y / a)\} f(a, b) \frac{d a d b}{a^{2}} .
\end{aligned}
$$

Compare (2.11). The right Fourier-Moyal transformation $\mathbb{F}_{\mathrm{M}}^{r}$ in turn is given by

$$
\begin{aligned}
\mathbb{F}_{\mathrm{M}}^{r}[f](z): & =\int \mathbb{E}_{R}(z, g) f(g) d_{r} g \\
& =\iint_{\text {aff }} \exp \{2 \pi i(-u x / y+v / y)\} f\left(e^{u}, v / \lambda(-u)\right) \frac{d u d v}{\lambda(-u)} \\
& =\iint_{\text {Aff }} \exp \{2 \pi i(-x \log a / y+b \lambda(\log a) / a y)\} f(a, b) \frac{d a d b}{a}
\end{aligned}
$$

We run a first few checks on these Fourier-Moyal kernels. For $a=1, b=0$, we recover trivially $\operatorname{Tr} \Omega^{L, R}(z)=1$. Also, from (7.3) for $g=1_{\text {Aff }}$ one gleans without effort the form (4.4) for the kernel of $\Omega(z)$, that was useful to invert the Wigner function in Section 4.5.

We should be able, as well, to recover the character from the right kernel, say. Indeed, since $\int_{ \pm \Pi} \Omega^{R}(z) d_{r} z=1 \mathcal{K}_{ \pm}$, the characters of the representations $U_{ \pm}$are retrieved from

$$
\int_{ \pm \Pi} e^{-2 \pi i x \log a / y} e^{2 \pi i b \lambda(\log a) / a y} \frac{d x d y}{y^{2}}=\int_{\mathbb{R}_{ \pm}^{\times}} \delta(a-1) e^{2 \pi i y b} \frac{d y}{|y|}=\chi_{ \pm}(g) .
$$

This leads to $\mathbb{F}_{\mathrm{M}}^{r}\left[\chi_{ \pm}\right]=\omega_{ \pm}$: the ugly duckling of a character (2.8) turns here into the swan of the symplectic form. The same holds for $\mathbb{F}_{\mathrm{M}}$. Also, the following equalities are immediate:

$$
\operatorname{Tr} U_{ \pm}[f]=\int_{ \pm \Pi} \mathbb{F}_{\mathrm{M}}[f](z) d_{l} z=\int_{ \pm \Pi} \mathbb{F}_{\mathrm{M}}^{r}[f](z) d_{r} z
$$

7.2. The modified Fourier-Moyal kernels. Just as the operator Fourier transform needs modification for groups that are not unimodular, we must redefine our Fourier kernels in order to get a Fourier inversion theorem and a Parseval formula. Consider now the following distribution or kernel:

$$
\mathbb{E}^{\bmod }(z, g):=\operatorname{Tr}\left(\Omega_{ \pm}(z) U_{ \pm}(g) d_{ \pm}^{1 / 2}\right)=W_{U_{ \pm}}(g) d_{ \pm}^{1 / 2}(z),
$$


for $z \in \pm \Pi$, respectively. Take $\operatorname{Im} z>0$. From (4.1), we obtain

$$
\Omega(z) U(g) d^{1 / 2}=y \iint_{\mathbb{R}^{2}} e^{-2 \pi i(x u+y v)} U\left(e^{u} a, e^{u}(b+v / \lambda(u)) M_{\sqrt{ } \cdot} d u d v,\right.
$$

and the kernel of this operator is thus

$$
\begin{aligned}
& \Omega U d^{1 / 2}(z, g ; r, s) \\
& \quad=y \iint_{\mathbb{R}^{2}} e^{-2 \pi i(x u+y v)} e^{2 \pi i e^{u}(b+v / \lambda(u)) r} s^{3 / 2} \delta\left(s-r e^{u} a\right) d u d v \\
& \quad=y \iint_{\mathbb{R}^{2}} e^{-2 \pi i(x u+y v)} s^{1 / 2} e^{2 \pi i e^{u}(b+v / \lambda(u)) r} \delta(u-\log (s / r a)) d u d v \\
& \quad=y \int_{\mathbb{R}} e^{-2 \pi i(x \log (s / r a)+y v)} e^{2 \pi i a^{-1} s(b+v / \lambda(\log (s / r a)))} \sqrt{s} d v \\
& =y e^{-2 \pi i x \log (s / r a)} e^{2 \pi i b s / a} \sqrt{s} \delta\left(y-\frac{s}{a \lambda(\log (s / r a))}\right) .
\end{aligned}
$$

Its trace gives us the desired kernel:

$$
\begin{aligned}
\mathbb{E}^{\bmod }(z, g) & =\int_{0}^{\infty} \Omega U d^{1 / 2}(z, g ; r, r) \frac{d r}{r} \\
& =y e^{2 \pi i x \log a} \int_{0}^{\infty} e^{2 \pi i r b / a} \delta(y-r / \lambda(\log a)) \frac{d r}{\sqrt{r}} \\
& =y e^{2 \pi i x \log a} \int_{0}^{\infty} e^{2 \pi i r b / a} \lambda(\log a) \delta(r-y \lambda(\log a)) \frac{d r}{\sqrt{r}} \\
& =\sqrt{|y| \lambda(\log a)} e^{2 \pi i(x \log a+y b \lambda(\log a) / a)} .
\end{aligned}
$$

We have written $|y|$ for $y$, so the formula remains valid on the second orbit. We then see that $\mathbb{E}^{\text {mod }}$ is no longer of modulus 1 . We check the coadjoint variation of this kernel, and find that

$$
\mathbb{E}^{\bmod }(z, g)=\Delta^{1 / 2}(h) \mathbb{E}^{\bmod }\left(h \triangleright z, h g h^{-1}\right) .
$$

In conclusion, we make the new definition

$$
\begin{aligned}
\mathbb{F}_{\mathrm{M}}^{\bmod }[f](z): & =\int \mathbb{E}^{\bmod }(z, g) f(g) d_{l} g \\
& =\sqrt{|y|} \iint_{\mathfrak{a f f}} \exp \{2 \pi i(u x+v y)\} f\left(e^{u}, v / \lambda(-u)\right) \frac{d u d v}{\sqrt{\lambda(u)}} .
\end{aligned}
$$

This is seen to coincide with the $\mathbb{F}_{\mathrm{AFK}}$ transform of [3]: compare (2.13).

Summarizing, the situation is as follows: the formulas analogous to (7.5) are true as well for the Fourier-Kirillov transform, as pointed out in Section 2; that is of 
course well known, and happens for the good reason that the character is concentrated at $a=1$ (i.e., at the subgroup generated by the Pukánszky subalgebra subordinate to the maximal orbits). On the other hand, for the inversion and Plancherel theorems to be perfect analogues of the ordinary case, we also need a modified Fourier-Moyal transform. This "fact of life" reflects the non-unimodularity of the group. The relevant results are established in the next section.

\subsection{The basic theorems of Fourier analysis}

Theorem 7.1. The left Fourier-Moyal kernel and transformation enjoy the following properties:

- Hermiticity: complex conjugation gives $\overline{\mathbb{E}(z, g)}=\mathbb{E}\left(z, g^{-1}\right)$.

- Covariance: $\mathbb{E}(z, g)=\mathbb{E}\left(h \triangleright z, h g h^{-1}\right)$ for all $h \in \mathrm{Aff}$.

- Character formula: $\int_{ \pm \Pi} \mathbb{E}(z, g) d \omega_{ \pm}(z)=\operatorname{Tr} U_{ \pm}(g)$.

- Convolution theorem: $\mathbb{F}_{\mathrm{M}}[f * h]=\mathbb{F}_{\mathrm{M}}[f] \star \mathbb{F}_{\mathrm{M}}[h]$.

Analogous properties hold for the right Fourier-Moyal kernel and map.

Proof. The first property is obvious from the definition and the selfadjointness of $\Omega(z)$. The second and third properties have already been established. The fourth is easy: note first that

$$
\mathbb{E}(\cdot, g) \star \mathbb{E}\left(\cdot, g^{\prime}\right)=\mathbb{E}\left(\cdot, g g^{\prime}\right)
$$

on account of (7.1) and (5.2). Therefore,

$$
\mathbb{F}_{\mathrm{M}}[f * h]=\iint_{\mathrm{Aff} \times \mathrm{Aff}} \mathbb{E}\left(\cdot, g g^{\prime}\right) f(g) h\left(g^{\prime}\right) d_{l} g d_{l} g^{\prime}=\mathbb{F}_{\mathrm{M}}[f] \star \mathbb{F}_{\mathrm{M}}[h] .
$$

Analogous properties hold for the modified kernel, as follows.

Theorem 7.2. The modified Fourier-Moyal kernel and transformation enjoy the following properties:

- Modified hermiticity: $\overline{\mathbb{E}^{\bmod }(z, g)}=\Delta^{-1 / 2}(g) \mathbb{E}^{\bmod }\left(z, g^{-1}\right)$.

- Modified covariance: $\mathbb{E}^{\bmod }(z, g)=\Delta^{1 / 2}(h) \mathbb{E}^{\bmod }\left(h \triangleright z, h g h^{-1}\right)$ for all $h \in$ Aff.

- Modified convolution: for all $f \in L^{1}$ (Aff, $d_{l} g$ ) and $h \in L^{1} \cap L^{2}$ (Aff, $d_{l} g$ ),

$$
\mathbb{F}_{\mathrm{M}}^{\bmod }(f * h)=\mathbb{F}_{\mathrm{M}}[f] \star \mathbb{F}_{\mathrm{M}}^{\bmod }[h]=\mathbb{F}_{\mathrm{M}}^{\bmod }[f] \star \mathbb{F}_{\mathrm{M}}\left[\Delta^{-1 / 2} h\right] .
$$

Proof. The first two are elementary. For the third, first notice that

$$
\mathbb{F}_{\mathrm{M}}[f](z)=\int_{\text {Aff }} \operatorname{Tr}\left(\Omega(z) U_{ \pm}(g)\right) f(g) d_{l} g=\operatorname{Tr}\left(\Omega(z) U_{ \pm}(f)\right)=W_{U_{ \pm}(f)}(z)
$$


for $z \in \pm \Pi$, when $f \in L^{1}$ (Aff, $d_{l} g$ ). On the other hand, when $h \in L^{1} \cap$ $L^{2}$ (Aff, $d_{l} g$ ), the relation

$$
U_{ \pm}(h) d_{ \pm}^{1 / 2}=d_{ \pm}^{1 / 2} U_{ \pm}\left(\Delta^{-1 / 2} h\right)
$$

follows from selfadjointness of $d$ and the semi-invariance relation (2.15), integrated over the group. More precisely, this equality on the domain of $d_{ \pm}^{1 / 2}$ extends to the operator closures, which are everywhere defined and Hilbert-Schmidt on $\mathcal{K}$ [3], [28]. Thus, over the orbits $\pm \Pi$ we obtain

$$
\begin{aligned}
\mathbb{F}_{\mathrm{M}}^{\bmod }(f * h) & =W_{U_{ \pm}(f) U_{ \pm}(h) d_{ \pm}^{1 / 2}=W_{U_{ \pm}}(f) \star W_{U_{ \pm}}(h) d_{ \pm}^{1 / 2}}=\mathbb{F}_{\mathrm{M}}[f] \star \mathbb{F}_{\mathrm{M}}^{\bmod }[h] \\
{[4 p t] } & =W_{U_{ \pm}(f) d_{ \pm}^{1 / 2} U_{ \pm}\left(\Delta^{-1 / 2} h\right)}=W_{U_{ \pm}(f) d_{ \pm}^{1 / 2} \star W_{U_{ \pm}}\left(\Delta^{-1 / 2} h\right)} \\
{[4 p t] } & =\mathbb{F}_{\mathrm{M}}^{\bmod }[f] \star \mathbb{F}_{\mathrm{M}}\left[\Delta^{-1 / 2} h\right] .
\end{aligned}
$$

The modifications make the nontrivial theorems of Fourier analysis available: namely, the analogue of the Schur orthogonality relations for compact groups (the $U_{ \pm}$ are discrete-series representations), the Fourier inversion theorem and the PlancherelParseval unitarity relation. (See [31] for the compact semisimple case and an application of it.) To state them, we extend the measure $\omega=\omega_{+} \cup \omega_{-}$from $\Pi \cup-\Pi$ to aff* $^{*}$ by declaring the complement $\mathbb{R}$ to be a nullset.

Theorem 7.3. These additional properties hold for the modified Fourier-Moyal kernel and transformation:

- Orthogonality: $\int_{\text {Aff }} \overline{\mathbb{E}^{\bmod }(z, g)} \mathbb{E}^{\bmod }(w, g) d_{l} g=|y| \delta(z-w)$ for $z, w \in \pm \Pi$.

- Inversion theorem: $f(g)=\int_{\mathfrak{a f f}^{*}} \overline{\mathbb{E}^{\bmod }(z, g)} \mathbb{F}_{\mathrm{M}}^{\bmod }[f](z) d \omega(z)$ whenever $f \in$ $\mathscr{D}($ Aff $)$.

- Plancherel formula: $\int_{\text {aff* }}\left|\mathbb{F}_{\mathrm{M}}^{\bmod }[f](z)\right|^{2} d \omega(z)=\int_{\mathrm{Aff}}|f(g)|^{2} d_{l} g$ whenever $f \in L^{2}\left(\right.$ Aff,$\left.d_{l} g\right)$.

Proof. For the Plancherel formula, it is enough to show the relation for $f \in \mathcal{D}$ (Aff); the extension to $L^{2}$ (Aff, $d_{l} g$ ) by unitarity is immediate.

Take $z=x+i y, w=u+i v$ in $\Pi$; the case of $z, w \in-\Pi$ is similar. The 
orthogonality relation is straightforward:

$$
\begin{aligned}
\int_{\text {Aff }} & \overline{\mathbb{E}^{\bmod }(z, g)} \mathbb{E}^{\bmod }(w, g) d_{l} g \\
& =\int_{\text {Aff }} \sqrt{y v} \lambda(\log a) e^{2 \pi i((x-u) \log a+b(v-y) \lambda(\log a) / a)} \frac{d a d b}{a^{2}} \\
& =\int_{0}^{\infty} \sqrt{y v} e^{2 \pi i(x-u) \log a} \delta(y-v) \frac{d a}{a}=|y| \delta(x-u) \delta(y-v) .
\end{aligned}
$$

The result obviously implies that

$$
\Omega(z)=\int_{\text {Aff }} \overline{\mathbb{E}^{\bmod }(z, g)} U(g) d^{1 / 2} d_{l} g,
$$

where the \pm signs have been omitted.

For the inversion formula, take $f \in \mathscr{D}$ (Aff); then

$$
\begin{aligned}
\int_{\text {aff }^{*}} & \overline{\mathbb{E}^{\bmod }(z, g)} \mathbb{F}_{\mathrm{M}}^{\bmod }[f](z) d \omega(z) \\
= & \int_{\text {aff }} \int_{\text {Aff }} \overline{\mathbb{E}^{\bmod }(z, g)} \mathbb{E}^{\bmod }\left(z, g^{\prime}\right) f\left(g^{\prime}\right) d_{l} g^{\prime} d \omega(z) \\
= & \int_{\Pi \cup-\Pi}|y| \int_{\mathrm{Aff}} \sqrt{\lambda(\log a)} \sqrt{\lambda\left(\log a^{\prime}\right)} \\
& e^{-2 \pi i\left(x\left(\log a-\log a^{\prime}\right)+y\left(b \lambda(\log a) / a-b^{\prime} \lambda\left(\log a^{\prime}\right) / a^{\prime}\right)\right)} f\left(a^{\prime}, b^{\prime}\right) \frac{d a^{\prime} d b^{\prime}}{a^{\prime 2}} \frac{d x d y}{y} \\
= & \int_{\mathbb{R}} \int_{\operatorname{Aff}} \lambda\left(\log a^{\prime}\right) \delta\left(a-a^{\prime}\right) e^{-2 \pi i y\left(b-b^{\prime}\right) \lambda\left(\log a^{\prime}\right) / a^{\prime}} f\left(a^{\prime}, b^{\prime}\right) \frac{d a^{\prime} d b^{\prime}}{a^{\prime}} d y \\
= & \int_{\mathbb{R}} \int_{\mathbb{R}} \frac{\lambda(\log a)}{a} e^{-2 \pi i y\left(b-b^{\prime}\right) \lambda(\log a) / a} f\left(a, b^{\prime}\right) d b^{\prime} d y=f(a, b) .
\end{aligned}
$$

We note the agreeable similarity between the Fourier transformation and cotransformation.

Finally, the Plancherel relation follows directly from the inversion theorem. Again we take $f \in \mathscr{D}$ (Aff), for simplicity:

$$
\begin{aligned}
\int_{\mathrm{aff}^{*}}\left|\mathbb{F}_{\mathrm{M}}^{\bmod }[f](z)\right|^{2} d \omega(z) & =\int_{\mathrm{aff} *} \int_{\mathrm{Aff}} \overline{f(g)} \overline{\mathbb{E}^{\bmod }(z, g)} \mathbb{F}_{\mathrm{M}}^{\bmod }[f](z) d_{l} g d \omega(z) \\
& =\int_{\mathrm{Aff}} \overline{f(g)} f(g) d_{l} g .
\end{aligned}
$$

We invite the reader to make a direct proof of this; it proceeds along the lines of the inversion formula, and is even shorter. 
Recall that in the case of a compact semisimple group, the Plancherel measure $d \omega(z)$ is supported on the integral coadjoint orbits $\mathcal{O}_{j}$, on each of which the formal dimension $d_{j}$ is a constant. By taking $\mathbb{F}_{\mathrm{M}}^{\bmod }(z, g):=d_{j}^{1 / 2} \mathbb{F}_{\mathrm{M}}(z, g)$ when $z \in \mathcal{O}_{j}$, one recovers the usual aspect of the Plancherel formula for $\|f\|^{2}$ as a weighted sum of integrals over these orbits [31]. The role of the Duflo-Moore operator as a formal dimension operator is transparent in our context.

Using Moore's concept of reduced character [60], defined for all $f \in \mathcal{D}$ (Aff), one can establish a character property for $\mathbb{F}_{M}$ mod . We forgo this. Finally, the right Fourier-Moyal kernel and transformation may be modified in the same way, leading to altogether analogous harmonic analysis properties, mutatis mutandis.

\section{Discussion}

\subsection{The Fronsdal program and differential equations for the Fourier-Moyal} kernel. In the terminology of [36], our $\mathbb{E}$-function is a $\star$-representation, that is, it satisfies equation (7.6). In view of the first part of theorem 7.1, we have a symmetric $\star$-representation (here called hermitian) in the sense of that reference. Such $\star$-representations are intrinsic objects on coadjoint orbit, introduced by Fronsdal as a (putative) lifting to the group level of the $\star$-exponentials of [9], which play a fundamental role in the theory of star products. They fulfil systems of differential equations. Concretely, Fronsdal's generic proposal for the $\star$-representation kernel is the locally given $\star$-exponential:

$$
\mathbb{E}_{\mathrm{F}}(F, g)=\exp _{\star}[2 \pi i X](F):=\sum_{n=0}^{\infty} \frac{(2 \pi i X)^{\star n}(F)}{n !}, \quad \text { if } g=e^{X} .
$$

The coefficient $2 \pi$ thrown in here is convenient, given our definitions. Good treatments of the $\star$-exponential are given by Arnal [5] and Gutt [48]. One readily sees that this object satisfies formally the equation of a $\star$-representation:

$$
\mathbb{E}_{\mathrm{F}}(\cdot, g) \star \mathbb{E}_{\mathrm{F}}\left(\cdot, g^{\prime}\right)=\mathbb{E}_{\mathrm{F}}\left(\cdot, g g^{\prime}\right) \text {. }
$$

From the covariance relation (8.1) one derives ordinary PDE for this type of $\star$-representation kernel, that may be sufficient to determine it under favourable circumstances. Substituting $e^{t X}$ for $g$ and $g^{\prime}$, for any $X \in \mathfrak{g}$ one obtains by differentiation of (8.1) at the formal level,

$$
\left[X, \mathbb{E}_{\mathrm{F}}\right]_{\star}=[r(X)-l(X)] \mathbb{E}_{\mathrm{F}},
$$

with $l(X), r(X)$ respectively the corresponding left- and right-invariant vector fields. We proceed now directly on aff and use its standard basis; then $X_{1} \equiv x, X_{2} \equiv y$. 
Thus because of the invariance formulae (5.6), we must have in our case:

$$
\begin{aligned}
& y \frac{\partial}{\partial y} \mathbb{E}(x, y ; a, b)=\left[r\left(X_{1}\right)-l\left(X_{1}\right)\right] \mathbb{E}(x, y ; a, b)=b \frac{\partial}{\partial b} \mathbb{E}(x, y ; a, b), \\
& y \frac{\partial}{\partial x} \mathbb{E}(x, y ; a, b)=\left[l\left(X_{2}\right)-r\left(X_{2}\right)\right] \mathbb{E}(x, y ; a, b)=(a-1) \frac{\partial}{\partial b} \mathbb{E}(x, y ; a, b) .
\end{aligned}
$$

This is the fundamental Fronsdal differential system for Aff. Direct inspection of the explicit form (7.4) of our $\mathbb{E}$ shows that these equations are indeed fulfilled. We already saw in Section 7.3 that the analogue of (8.1) is satisfied by our $\mathbb{E}$ as well.

Following the Fronsdal program, invariant affine $\star$-quantization was studied in [51]; the latter is the oldest work on quantization based on the affine group of which we are aware. Equations (8.2) coincide with equations (2.9) of [51], when allowance is made for a slightly different definition of the affine group multiplication. Of course, our focus in this paper is on the tracial property rather than general covariance. Thus we did obtain a distinguished solution.

8.2. Relation with the formalism of Ali et al. In [2], [3], [54] taken in the context of the affine group of the line, the Wigner functions are indirectly defined as the images of the map

$$
\operatorname{HS}\left(\mathcal{K}_{+}\right) \oplus \operatorname{HS}\left(\mathcal{K}_{-}\right) \rightarrow L^{2}\left(\mathrm{aff}^{*}, d \omega_{+} \cup d \omega_{-}\right),
$$

obtained by composition of the inverse Plancherel transformation and the $\mathbb{F}_{\mathrm{AFK}}$-transformation already given in Section 2.3:

$$
W[A]=\mathbb{F}_{\mathrm{AFK}}\left[P^{-1}(A)\right] .
$$

These authors furthermore propose "formal Wigner operators" $W(F)$ via the property

$$
\operatorname{Tr}(A W(F)):=W[A](F) .
$$

In view of the results in Section 7.3, it is clear that the dequantization $W_{A}$ in (1.1) and in Section 4.5 is the same as $W[A]$, and the formal Wigner operators $W(F)$ are just the Stratonovich-Weyl (de)quantizers $\Omega(F)$, which are not merely formal at all, and have been explicitly calculated in this paper. It is remarkable that the integral expression (49) for $W(F)$ in [2] makes manifest use of the Duflo-Moore operators, whereas ours does not; they however coincide.

Nevertheless, the difference between our axiomatic approach and the treatment based on the Plancherel transform and the a priori Fourier transformation (2.12) is not moot. Only the coadjoint orbits have an interpretation as elementary physical systems (see [19] in this respect); the coalgebra $\mathrm{g}^{*}$ by itself is an empty vessel. Now, the second definition raises the problem of the eventual indecomposability of $W[A]$ on the coadjoint orbits; or, on account of the Kirillov map, on the unirreps. This indecomposability actually happens [3]. Moreover the definition of $\mathbb{F}_{\mathrm{AFK}}$ will not do 
for compact groups; and the character formula à la Kirillov is lost with it, anyway. It seems preferable to accept that the Stratonovich-Weyl quantizer generally determines the correct scalar Fourier transform, rather than the other way around, and that for a non-unimodular group there are four such pertinent objects: $\mathbb{F}_{\mathrm{M}}, \mathbb{F}_{\mathrm{M}}^{\bmod }$ and $\mathbb{F}_{\mathrm{M}}^{r}, \mathbb{F}_{\mathrm{M}}^{\mathrm{mod}, r}$.

In other words, our approach is geared to fit better with Kirillov theory. It has an obvious drawback, in that no one knows precisely for which categories of groups and unirreps do quantizers exist (for non-type-I groups there is no hope whatsoever). We give an aperçu of the question in the following section, through the story of Stratonovich-Weyl operators so far.

To conclude, note that our Weyl Ansatz for $Q(f)$ can be rewritten in the form

$$
Q(f)=\int_{\text {aff }} F[f](u, v) U_{ \pm}\left(e^{u}, v e^{u} / \lambda(u)\right) d u d v
$$

where $F$ is the ordinary Fourier transformation between functions on aff* and on aff, and to use it, we extend $f$ by zero on the complement of $\Pi$. Thus, the quantization prescription is not unrelated to the proposal of Manchon [56] for Weyl quantization of solvable Lie groups - which however ignores the issue of supports within coadjoint orbits, needed to establish boundedness or compactness of the quantized operators.

8.3. Setting the record straight. The concept of Stratonovich-Weyl quantizer was introduced in the late eighties [45], [77] by two of us. In [46], Section 3.5, we reported that the name had not caught on, and called them "Moyal quantizers" instead. But the concept itself certainly did catch on, and beyond [15], which inaugurated a wealth of applications, we find it in [2] under the name "Wigner operators". Lest that nomenclature be misread as a priority claim, it seems wise to revert to form. We still speak here of Moyal-type quantization for tracial quantization, and of Fourier-Moyal kernels and transformations.

The main motivation for the early works was to extend the remit of phase-space Quantum Mechanics. In particular, tracial twisted products covariant under SU(2), for dealing with spinning particles, were developed in full detail, including applications, in [77]. There we were elaborating on old work by Stratonovich [73] - who should thus be credited with introducing the "fuzzy sphere" - and were unaware of another precedent [1]. An equivalent version of the SU(2)-Stratonovich-Weyl quantizer, simpler than our original expression, is given in [49].

The Stratonovich-Weyl quantizer appropriate to deal with relativistic particles [19] was developed shortly after [45], [77]. Indeed, the prevalence of the Heisenberg groups in quantization is an artifact. From the physical viewpoint, the coadjoint orbit for the 7-dimensional Heisenberg group makes its appearance as a direct factor of the splitting group $\widetilde{\mathrm{Gal}}$ of the covering group of the Galilei group [17], that linearizes its multiplier representations. Thus the restriction of the quantizer for $\widetilde{\mathrm{Gal}}$ to the flat part of the orbits renders the standard Moyal quantizer [45]; for an explicit calcula- 
tion showing the multiplier Galilean covariance of the ordinary Moyal framework, peruse [65]. All this often goes unremarked.

Stratonovich-Weyl quantizers exist for all compact Lie groups. This was shown in principle in [31] by the time-honoured method of interpolating between the "active" and "passive" symbols associated to semitracial quantizers. Then in the nineties, apparently unaware of that work, N. V. Pedersen introduced a similar set of postulates, and proved the existence of Stratonovich-Weyl quantizers, for nilpotent Lie groups [64]. See also [37], Section 4.5, on this matter. Prior to all that, the Unterbergers [76] had shown by the interpolation method the existence of a Moyal-type quantization for the discrete-series representations of $\operatorname{SL}(2, \mathbb{R})$. In this regard, we wish to mention [66] as well. (To our knowledge, however, no one has been able to exhibit explicitly the Stratonovich-Weyl quantizers for this case.) These older examples and the work of Ali and coworkers indicate that Stratonovich-Weyl quantizers exist for large classes of semi-direct product groups. The time seems ripe for a renewed assault on Moyal-type quantization and scalar group Fourier transforms covariant under larger classes of solvable and reductive groups.

Fourier-Moyal kernels are arguably even more important than Stratonovich-Weyl quantizers, because of their crucial role in harmonic analysis. They seem destined to complete Kirillov theory. For years, the abstract nature of expansions of functions on Lie groups in terms of equivalence classes of unitary transformations has been a source of some dissatisfaction [50]. However, one still finds the Plancherel measure usually realized on $\widehat{G}$, rather than on $\mathrm{g}^{*}$. For compact group symmetry, we demonstrated in [31] how the Fourier-Moyal transformation solves the problem of giving a formulation of harmonic analysis parallel to standard Fourier analysis. This section and the previous one show a wider applicability of its method; and, although here we have opted for concrete proofs, there is a good chance, in view of the Leptin-Ludwig theorem, that similar results are valid for all exponential groups. To finish, we should mention that a bit earlier - see [6] and references therein - a concept of scalar "adapted Fourier transform" had been proposed; because of covariance trouble it actually does not seem to be all that well adapted to the context.

\section{Spectral triples on the half-plane}

We turn at last to noncommutative spectral triples. The upper half-plane $\Pi$ is a model for the simplest hyperbolic geometry, living on a Riemannian surface with negative constant scalar curvature. It may be regarded as a homogeneous space of the group $\operatorname{SL}(2, \mathbb{R})$, acting by Möbius transformations $z \mapsto(a z+b) /(c z+d)$ on $\Pi$.

Writing a typical element of the Iwasawa decomposition $\operatorname{SL}(2, \mathbb{R})=A N K$ as

$$
g=a_{t} n_{s} k_{\theta}=\left(\begin{array}{cc}
e^{t / 2} & 0 \\
0 & e^{-t / 2}
\end{array}\right)\left(\begin{array}{ll}
1 & s \\
0 & 1
\end{array}\right)\left(\begin{array}{cc}
\cos \frac{1}{2} \theta & \sin \frac{1}{2} \theta \\
-\sin \frac{1}{2} \theta & \cos \frac{1}{2} \theta
\end{array}\right),
$$


the compact subgroup $K=\mathrm{SO}(2)$ fixes $i$ and thus $\Pi \approx \mathrm{SL}(2, \mathbb{R}) / \mathrm{SO}(2)$ is a principal homogeneous space for the subgroup $A N$. The orbits of $A$ are half-lines emanating from 0 , and the orbits of $N$ are horizontal lines: see Figure 3.

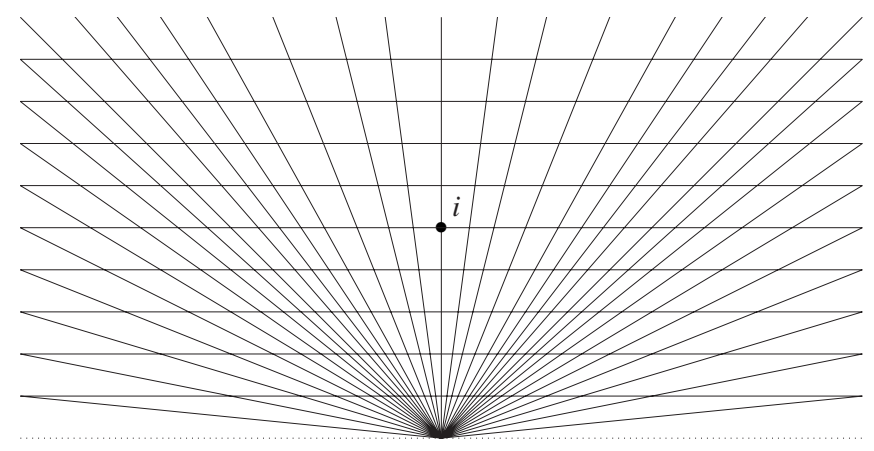

Figure 3. Orbits of $A$ and $N$ for the half-plane.

Under the identification

$$
A N \ni a_{t} n_{s} \longleftrightarrow a_{t} n_{s} \cdot i=a_{t} \cdot(i+s)=s e^{t}+i e^{t} \equiv x+i y=z \in \Pi,
$$

we may transfer the group operation of $A N$ to $\Pi$ by letting

$$
(x+i y) \cdot\left(x^{\prime}+i y^{\prime}\right):=x+x^{\prime} y+i y y^{\prime} .
$$

On the other hand, we may identify $A N$ with Aff by the group isomorphism

$$
a_{t} n_{s} \longmapsto\left(\begin{array}{cc}
e^{t} & -s e^{t} \\
0 & 1
\end{array}\right)
$$

Observe, however, that the product $\bullet$ induced by the (left) Möbius action of Aff on $\Pi$ is opposite to that of (2.7), induced by the (left) coadjoint action of Aff on $\Pi$. This is to be expected, since (9.1) and the identification (9.2) leads to $(a, b)=(y,-x)$, which is $g_{z}^{-1}$ with the definition (2.6).

The spinor bundle $S \rightarrow \Pi$ over the Poincare half-plane has rank two and is the direct sum of two trivial line bundles, since $\Pi$ is contractible. Let $\mathscr{H}_{0}:=$ $L^{2}\left(\Pi, y^{-2} d x d y\right)$ and let $\mathscr{H}:=\mathscr{H}_{0} \oplus \mathscr{H}_{0}$ be the Hilbert space of spinors. The Dirac operator is $\not D:=-i\left(\sigma_{+} \nabla_{E_{+}}^{S}+\sigma_{-} \nabla_{E_{-}}^{S}\right)$, using the isotropic basis $E_{+}:=$ $2 y \partial_{z}=y\left(\partial_{x}-i \partial_{y}\right), E_{-}:=2 y \bar{\partial}_{z}=y\left(\partial_{x}+i \partial_{y}\right)$, and the spin connection $\nabla_{E_{\rho}}^{S}:=E_{\rho}-\frac{1}{4} \widehat{\Gamma}_{\rho \alpha}^{\beta} \sigma^{\alpha} \sigma_{\beta}$ is determined by the Christoffel symbols of the LeviCivita connection, for any zweibein $\left\{E_{\rho}\right\}$. The Levi-Civita connection is canonical here because $\Pi$ is a symmetric space [68]. Standard formulas [46], [62] then yield

$$
\not D=-i\left(\begin{array}{cc}
0 & 2 y \partial_{z}+\frac{i}{2} \\
2 y \bar{\partial}_{z}-\frac{i}{2} & 0
\end{array}\right) \text {. }
$$


Following Palmer et al [63], one can find a representation $\tau$ of $\operatorname{SL}(2, \mathbb{R})$ on $\mathscr{H}$ under which $\not D$ is invariant. It can be written in the form

$$
[\tau(g) \psi](z):=u(g, z) \psi\left(g^{-1} \cdot z\right),
$$

where the factor $u$ is of the form

$$
u(g, z):=\left(\begin{array}{cc}
v\left(g^{-1}, z\right)^{1 / 2} & 0 \\
0 & v\left(g^{-1}, z\right)^{-1 / 2}
\end{array}\right),
$$

with $v(g, z):=\frac{c \bar{z}+d}{c z+d}$ for $g=\left(\begin{array}{ll}a & b \\ c & d\end{array}\right)$, whenever the square root of $z \mapsto v(g, z)$ can be chosen smoothly, e.g., for $g$ lying in suitable one-parameter subgroups. A set of infinitesimal generators $F_{0}, F_{1}, F_{2}$ representing $\mathfrak{s}(2, \mathbb{R})$ is found to be

$$
\begin{aligned}
& F_{0}=-\frac{1}{2}\left(1+z^{2}\right) \partial_{z}-\frac{1}{2}\left(1-\bar{z}^{2}\right) \bar{\partial}_{z}+\frac{1}{4}(z-\bar{z}) \sigma_{3}, \\
& F_{1}=-\left(z \partial_{z}+\bar{z} \bar{\partial}_{z}\right)=-\left(x \partial_{x}+y \partial_{y}\right), \quad F_{0}+F_{2}=-\partial_{x} .
\end{aligned}
$$

The invariance of $\not D$ follows directly from the relations $\left[F_{j}, \not D\right]=0$. For instance, $\left[F_{1}, \not D\right]$ vanishes because $\left[x \partial_{x}+y \partial_{y}, y \partial_{x} \mp i y \partial_{y}\right]=0$. One can observe that the components of $\not D$ are left-invariant differential operators on $\Pi$, regarded as a group. This is why they commute with the fundamental vector fields $F_{1}$ and $F_{0}+F_{2}$, which are of course right-invariant [18].

Now let a suitably chosen algebra of functions on $\Pi$, under the twisted product, act diagonally on spinors. This defines on $\Pi$ a noncommutative operator module in the sense of [8]. That is to say, there is a noncommutative algebra $(\mathcal{A}, \star)$ involutively represented by bounded operators on a Hilbert space, and a self-adjoint operator $D$ on the same Hilbert space, unbounded in the present case, whose domain is preserved by the action of $\mathcal{A}$.

We shall now show that the basic pre-condition for a spectral triple holds, to wit, the commutator of $D$ with the twisted multiplication by elements of $\mathcal{A}$ is bounded.

The expression (5.3) may be applied componentwise when the function $h$ is replaced by the two-spinor $\phi$, namely:

$$
R_{w} \phi(z)=\left(\begin{array}{l}
\phi_{1}(z \cdot w) \\
\phi_{2}(z \cdot w)
\end{array}\right)=\left(\begin{array}{l}
\phi_{1}(w \cdot z) \\
\phi_{2}(w \cdot z)
\end{array}\right)=: L_{w}^{\cdot} \phi(z),
$$

with an obvious notation. Now, because the factor $u(g, z)$ is trivial for $g \in A N$, the invariance of $\not D$ under all $\tau(g)$ allows us to conclude that $L_{w}^{\cdot} \not D=\not D L_{w}^{*}$, and thus

$$
\begin{aligned}
& \not D(f \star \phi)(z)-f \star \not D \phi(z) \\
& \quad=\iint_{\Pi^{2}} K_{\star}(i, w, t)\left(\not D_{z}\left(L_{w}^{\cdot} f(z) L_{t}^{\cdot} \phi(z)\right)-L_{w}^{\cdot} f(z) \not D_{z} L_{t}^{\cdot} \phi(z)\right) d_{l} w d_{l} t \\
& \quad=\iint_{\Pi^{2}} K_{\star}(i, w, t) L_{w}^{\cdot} \not D f(z) L_{t}^{\cdot} \phi(z) d_{l} w d_{l} t=\not D f \star \phi(z),
\end{aligned}
$$


where the second equality uses the invariance of the Dirac operator under the (restriction to $A N$ of) the $\operatorname{SL}(2, \mathbb{R})$-action by Möbius transformations on its orbit $\Pi$. Thus the Leibniz rule is valid for the action of $\not D$ on the (left-covariant for the coadjoint action, right-covariant for the Möbius action) twisted product. In particular, if $L_{\star}(f)$ denotes the operator of left twisted multiplication, then $\left[\not D, L_{\star}(f)\right]=L_{\star}(\not D f)$ is bounded whenever $f$ has a bounded exterior derivative. Moreover, arguing as in [38], we also get Connes' first-order condition [21] from the associativity and complex conjugation properties of the twisted product. Needless to say, from here to showing or disproving that the Moyal half-plane in our sense is a noncommutative geometry, there is still some way to travel.

Some reflection on what has been achieved - and what has not - is in order. The half-plane carries a natural symmetry, namely the left Möbius action of SL(2, R). However, in order to preserve the Leibniz rule, we have been led to an algebra which is right-invariant, rather than left-invariant, under (the restriction to the subgroup $A N$ of) that action. To our knowledge, this was first stated in [14], which moreover contains a beautiful study of the differential equations a general covariant trikernel must satisfy. If one insists on having left-invariance of the algebra under the Möbius action, one can bring into play instead the Dirac operator associated to the right-invariant metric on $A N$, which is given by

$$
Q=-i\left(\begin{array}{cc}
0 & (x-i) \partial_{x}+y \partial_{y}+\frac{1}{2} \\
(x+i) \partial_{x}+y \partial_{y}+\frac{1}{2} & 0
\end{array}\right) .
$$

Alternatively, one might try to deform the Dirac operator itself.

\section{Outlook}

We conclude with a brief review of possible ramifications for our work in this paper.

- Harmonic analysis by way of the scalar Fourier kernels can of course be pursued much further, around standard lines. For instance, the third assertion in Theorem 7.2 remains true when $f$ is a bounded measure. In general, one uses the power $d_{ \pm}^{1 / p}$ of the dimension operators for $L^{p}$-Fourier analysis. Some matters of rigour - see the remark at the end of Section 4.5 - will be treated separately [41]. More to the point, the role of the Fourier-Moyal transformation in relating Wildberger's group class and coadjoint orbit hypergroups [83], arguably in the spirit of [35], is an appealing subject of research. See, with regard to Wildberger's theory, the remarks in [53, Section 6.4].

- The issue of $\operatorname{SL}(2, \mathbb{R})$ symmetry for a satisfactory star product is not ended. It should be obvious that $K_{\star}^{R}$ can be extended to define semitracial, $\operatorname{SL}(2, \mathbb{R})$-covariant star products on the half-plane. That should allow a fresh attack on the 
determination of the Stratonovich-Weyl quantizers for this group [13]. A solution would bring the prize of a suitable and strong definition of noncommutative Riemann surfaces.

- There seems to be no obstruction to the generalization of our method for constructing star products on $A N$-symmetric spaces, on the basis of the Iwasawa decomposition. For complex groups, this leads naturally to Manin triples - see for instance [16]. ${ }^{3}$

Acknowledgments. Most of this work was done at Universidad Complutense of Madrid (UCM), at a period when the second named author was a staff member there. We are thankful for helpful discussions to Paolo Aniello, Pierre Bieliavsky, Alain Connes, Bruno Iochum, Fedele Lizzi, Giuseppe Marmo, Patrick Várilly, Jasson Vindas, and Patrizia Vitale. Special thanks are due to André Unterberger, who shared with us his unpublished manuscript [75], after the first version of our article was written. VG thanks the UCM for hospitality. JMG-B acknowledges partial support from CICyT, Spain, through the grant FIS2005-02309, and is grateful to Università di Napoli Federico II for warm hospitality. JCV is grateful to UCM and to Banco Santander for a Visitante Distinguido fellowship, and thanks the Universidad de Zaragoza and SISSA, Trieste, for friendly hospitality. Support for JCV from the Universidad de Costa Rica is acknowledged. We also thank the referee, whose comments helped to improve the final manuscript.

\section{References}

[1] G. S. Agarwal, Relation between atomic coherent-state representation, state multipoles, and generalized phase-space distributions. Phys. Rev. A 24 (1981), 2889-2896. MR 639401

[2] S. T. Ali, N. M. Atakishiyev, S. M. Chumakov, and K. B. Wolf, The Wigner function for general Lie groups and the wavelet transform. Ann. Henri Poincaré 1 (2000), 685-714. Zbl 1024.81015 MR 1785185

[3] S. T. Ali, H. Führ, and A. E. Krasowska, Plancherel inversion as unified approach to wavelet transforms and Wigner functions. Ann. Henri Poincaré 4 (2003), 1015-1050. Zbl 1049.81043 MR 2031158

[4] P. Aniello, Square integrable projective representations and square integrable representations modulo a relatively central subgroup. Internat. J. Geom. Methods Mod. Phys. 3 (2006), 233-267. Zbl 1088.22002 MR 2213819

[5] D. Arnal, The *-exponential. In Quantum theories and geometry (Les Treilles, 1987), Math. Phys. Stud. 10, Kluwer Acad. Publ., Dordrecht 1988, 23-51. MR 0976863

\footnotetext{
${ }^{3}$ Patrizia Vitale pointed this out to us.
} 
[6] D. Arnal and J. C. Cortet, Représentations * des groupes exponentiels. J. Funct. Anal. 92 (1990), 103-135. Zbl 0726.22011 MR 1064689

[7] K. Banaszek and K. Wódkiewicz, Direct probing of quantum phase space by photon counting. Phys. Rev. Lett. 76 (1996), 4344-4347.

[8] C. Bär, Conformal structures in noncommutative geometry. J. Noncommut. Geom. 1 (2007), 385-395. MR 2314101

[9] F. Bayen, M. Flato, C. Fronsdal, A. Lichnerowicz, and D. Sternheimer, Deformation theory and quantization. I. Deformations of symplectic structures. Ann. Physics 111 (1978), 61-110. Zbl 0377.53024 MR 0496157

[10] J. Bertrand and P. Bertrand, A class of affine Wigner functions with extended covariance properties. J. Math. Phys. 33 (1992), 2515-2527. Zbl 0787.22022 MR 1167952

[11] J. Bertrand and P. Bertrand, Symbolic calculus on the time-frequency half-plane. J. Math. Phys. 39 (1998), 4071-4090. Zbl 0931.94003 MR 1633195

[12] P. Bieliavsky, Strict quantization of solvable symmetric spaces. J. Symplectic Geom. 1 (2002), 269-320. Zbl 1032.53080 MR 1959584

[13] P. Bieliavsky, V. Gayral, and B. Iochum. In preparation.

[14] P. Bieliavsky, S. Detournay, P. Spindel, and M. Rooman, Star products on extended massive non-rotating BTZ black holes. J. High Energy Phys. 06 (2004), 031. MR 2084424

[15] C. Brif and A. Mann, A general theory of phase-space quasiprobability distributions. J. Phys. A 31 (1998), L9-L17. Zbl 0956.81030 MR 1620276

[16] M. Cahen, S. Gutt, and J. Rawnsley, Some remarks on the classification of Poisson Lie groups. In Symplectic geometry and quantization (Sanda and Yokohama, 1993), Contemp. Math. 179, Amer. Math. Soc., Providence, RI, 1994, 1-16. Zbl 0820.58018 MR 1319599

[17] J. F. Cariñena, M. A. Del Olmo, and M. Santander, Locally operating realizations of transformation Lie groups. J. Math. Phys. 26 (1985), 2096-2106. Zbl 0575.22019 MR 801096

[18] J. F. Cariñena, K. Ebrahimi-Fard, H. Figueroa, and J. M. Gracia-Bondía, Hopf algebras in dynamical systems theory. Int. J. Geom. Methods Mod. Phys. 4 (2007), 577-646. Zbl 05277109 MR 2343429

[19] J. F. Cariñena, J. M. Gracia-Bondía, and J. C. Várilly, Relativistic quantum kinematics in the Moyal representation. J. Phys. A 23 (1990), 901-933. Zbl 0706.60108 MR 1048769

[20] A. Connes, La notion de variété et les axiomes de la géométrie. Course at the Collège de France, January-March 1996.

[21] A. Connes, Gravity coupled with matter and the foundation of non-commutative geometry. Comm. Math. Phys. 182 (1996), 155-176. Zbl 0881.58009 MR 1441908

[22] A. Connes, M. Flato, and D. Sternheimer, Closed star products and cyclic cohomology. Lett. Math. Phys. 24 (1992), 1-12. Zbl 0767.55005 MR 1162894

[23] A. Connes and H. Moscovici, The $L^{2}$-index theorem for homogeneous spaces of Lie groups. Ann. of Math. (2) 115 (1982), 291-330. Zbl 0515.58031 MR 647808

[24] S. Detournay, Deformations of anti-de Sitter black holes. Ph. D. thesis, Université de Mons-Hainaut, Mons 2006; arXiv:hep-th/0611031v1 
[25] J. Dixmier, L'application exponentielle dans les groupes de Lie résolubles. Bull. Soc. Math. France 85 (1957), 113-121. Zbl 0077.25203 MR 0092930

[26] J. Dixmier, Les $C^{*}$-algèbres et leurs représentations. Deuxième édition, Cahiers Scientifiques, Fasc. XXIX, Gauthier-Villars, Paris 1969. Zbl 0174.18601 MR 0246136

[27] M. Duflo, Caractères des groupes et des algèbres de Lie résolubles. Ann. Sci. École Norm. Sup. (4) 3 (1970), 23-74. Zbl 0223.22016 MR 0269777

[28] M. Duflo and C. C. Moore, On the regular representation of a nonunimodular locally compact group. J. Funct. Anal. 21 (1976), 209-243. Zbl 0317.43013 MR 0393335

[29] J. M. G. Fell and R. S. Doran, Representations of *-algebras, locally compact groups, and Banach *-algebraic bundles. Pure Appl. Math. 125, 126, Academic Press, Boston 1988. Zbl 0652.46050 Zbl 0652.46051 MR 0936628 MR 0936629

[30] H. Figueroa and J. M. Gracia-Bondía, Combinatorial Hopf algebras in quantum field theory I. Rev. Math. Phys. 17 (2005), 881-976. Zbl 1090.16016 MR 2167639

[31] H. Figueroa, J. M. Gracia-Bondía, and J. C. Várilly, Moyal quantization with compact symmetry groups and noncommutative harmonic analysis. J. Math. Phys. 31 (1990), 2664-2671. Zbl 0753.43002 MR 1075750

[32] T. Filk, Divergencies in a field theory on quantum space. Phys. Lett. B 376 (1996), 53-58. MR 1395558

[33] P. Flandrin, Separability, positivity, and minimum uncertainty in time-frequency energy distributions. J. Math. Phys. 39 (1998), 4016-4040. Zbl 0928.94004 MR 1633203

[34] P. Flandrin and P. Gonçalvès, Geometry of affine time-frequency distributions. Appl. Comput. Harmon. Anal. 3 (1996), 10-39. Zbl 0852.94001 MR 1374393

[35] F. G. Frobenius, Über Gruppencharaktere. Sitzungsber. Preuß. Akad. Wiss. Berlin (1896), 985-1021; reprinted in Gesammelte Abhandlungen, vol. III, Springer-Verlag, Berlin 1968, 985-1021. JFM 27.0092.01

[36] C. Fronsdal, Some ideas about quantization. Rep. Math. Phys. 15 (1979), 111-145. Zbl 0418.58011 MR 551133

[37] H. Führ, Abstract harmonic analysis of continuous wavelet transforms. Lecture Notes in Math. 1863, Springer-Verlag, Berlin 2005. Zbl 1060.43002 MR 2130226

[38] V. Gayral, J. M. Gracia-Bondía, B. Iochum, T. Schücker, and J. C. Várilly, Moyal planes are spectral triples. Comm. Math. Phys. 246 (2004), 569-623. Zbl 1084.58008 MR 2053945

[39] V. Gayral, J. M. Gracia-Bondía, and F. Ruiz Ruiz, Position-dependent noncommutative products: classical construction and field theory. Nuclear Phys. B 727 (2005), 513-536. Zbl 1126.81311 MR 2173731

[40] V. Gayral, B. Iochum, and J. C. Várilly, Dixmier traces on noncompact isospectral deformations. J. Funct. Anal. 237 (2006), 507-539. Zbl 1106.46054 MR 2230348

[41] V. Gayral and J. C. Várilly, Operator-valued distributions in harmonic analysis and phasespace quantum mechanics. J. Generalized Functions, in preparation.

[42] J. M. Gracia-Bondía, Generalized Moyal quantization on homogeneous symplectic spaces. In Deformation theory and quantum groups with applications to mathematical physics (Amherst, MA, 1990), Contemp. Math. 134, Amer. Math. Soc., Providence, RI 1992, 93-114. Zbl 0788.58024 MR 1187280 
[43] J. M. Gracia-Bondía, F. Lizzi, G. Marmo, and P. Vitale, Infinitely many star products to play with. J. High Energy Phys. 04 (2002), 026. MR 1911415

[44] J. M. Gracia-Bondía and J. C. Várilly, Algebras of distributions suitable for phase-space quantum mechanics. I. J. Math. Phys. 29 (1988), 869-879. Zbl 0652.46026 MR 940351

[45] J. M. Gracia-Bondía and J. C. Várilly, Phase-space representation for Galilean quantum particles of arbitrary spin. J. Phys. A 21 (1988), L879-L883. Zbl 0653.46070 MR 975152

[46] J. M. Gracia-Bondía, J. C. Várilly, and H. Figueroa, Elements of noncommutative geometry. Birkhäuser Adv. Texts, Birkhäuser, Boston 2001. Zbl 0958.46039 MR 1789831

[47] H. J. Groenewold, On the principles of elementary quantum mechanics. Physica 12 (1946), 405-460. Zbl 0060.45002 MR 0018562

[48] S. Gutt, Some aspects of deformation theory and quantization. In Quantum theories and geometry (Les Treilles, 1987), Math. Phys. Stud. 10, Kluwer Academic Publishers, Dordrecht 1988, 77-102. MR 0976866

[49] S. Heiss and S. Weigert, Discrete Moyal-type representations for a spin. Phys. Rev. A 63 (2001), 012105. MR 1816608

[50] S. Helgason, The Fourier transform on symmetric spaces. In Élie Cartan et les mathématiques d'aujourd'hui (Lyon, 1984), Astérisque 1985, numéro hors série, 151-164. Zbl 0611.43005 MR 837200

[51] T. V. Huynh, Invariant *-quantization associated with the affine group. J. Math. Phys. 23 (1982), 1082-1087. MR 660012

[52] I. Khalil, Sur l'analyse harmonique du groupe affine de la droite. Studia Math. 51 (1974), 139-167. Zbl 0294.43007 MR 0350330

[53] A. A. Kirillov, Lectures on the orbit method. Grad. Stud. Math. 64, Amer. Math. Soc., Providence, RI, 2004. Zbl 02121486 MR 2069175

[54] A. E. Krasowska and S. T. Ali, Wigner functions for a class of semi-direct product groups, J. Phys. A 36 (2003), 2801-2820. Zbl 1069.81550 MR 1965293

[55] H. Leptin and J. Ludwig, Unitary representation theory of exponential Lie groups. de Gruyter Exp. Math. 18, Walter de Gruyter \& Co., Berlin 1994. Zbl 0833.22012 MR 1307383

[56] D. Manchon, Weyl symbolic calculus on any Lie group. Acta Appl. Math. 30 (1993), 159-186. Zbl 0779.22005 MR 1204731

[57] C. Marquardt, J. Heersink, R. Dong, M. V. Chekhova, A. B. Klimov, L. L. SánchezSoto, U. L. Andersen, and G. Leuchs, Quantum reconstruction of an intense polarization squeezed optical state. Phys. Rev. Lett. 99 (2007), 220401. Zbl MR

[58] O. Mathieu, Bicontinuity of the Dixmier map. J. Amer. Math. Soc. 4 (1991), 837-863. Zbl 0743.17013 MR 1115787

[59] B. Molnár, M. G. Benedict, and J. Bertrand, Coherent states and the role of the affine group in the quantum mechanics of the Morse potential. J. Phys. A 34 (2001), 3139-3151. Zbl 0998.81034 MR 1832779 
[60] C. C. Moore, Representations of solvable and nilpotent groups and harmonic analysis on nil and solvmanifolds. In Harmonic analysis on homogeneous spaces (Williams Coll., Williamstown, Mass., 1972), Proc. Sympos. Pure Math. 26, Amer. Math. Soc., Providence, RI, 1973, 3-44. Zbl 0292.22015 MR 0385001

[61] J. E. Moyal, Quantum mechanics as a statistical theory. Proc. Cambridge Philos. Soc. 45 (1949), 99-124. Zbl 0031.33601 MR 0029330

[62] M. Nakahara, Geometry, topology and physics. 2nd ed., Grad. Stud. Ser. Phys., Institute of Physics, Bristol 2003. Zbl 1090.53001 MR 2001829

[63] J. Palmer, M. Beatty, and C. A. Tracy, Tau functions for the Dirac operator on the Poincaré disk. Comm. Math. Phys. 165 (1994), 97-173. Zbl 0812.35112 MR 1298945

[64] N. V. Pedersen, Matrix coefficients and a Weyl correspondence for nilpotent Lie groups. Invent. Math. 118 (1994), 1-36. Zbl 0848.22016 MR 1288465

[65] J. F. Plebański, M. Przanowski, J. Tosiek, and F. J. Turrubiates, Remarks on deformation quantization on the cylinder. Acta Phys. Polon. B 31 (2000), 561-587. Zbl 1010.53066 MR 1753753

[66] O. Protti, Cuantización sobre el disco de Poincaré. San José 1989, unpublished; J. C. Várilly, Teoría de grupos en cuantización. CINVESTAV del IPN, México, DF, 1992.

[67] A. Rennie, Smoothness and locality for nonunital spectral triples. K-Theory 28 (2003), 127-165. Zbl 1027.46088 MR 1995874

[68] M. A. Rieffel, A global view of equivariant vector bundles and dirac operators on some compact homogeneous spaces. In Group representations, ergodic theory, and mathematical physics, Contemp. Math. 449, Amer. Math. Soc., Providence, RI, 2008, 399-415. Zbl MR

[69] P. de M. Rios and G. M. Tuynman, On Weyl quantization from geometric quantization. Preprint 2002. arXiv:math-ph/0201044v2

[70] W. Rossmann, Some toric manifolds and a path integral. In The orbit method in geometry and physics (Marseille, 2000), Progr. Math. 213, Birkhäuser, Boston 2003, 395-419. Zbl 1076.53112 MR 1995386

[71] A. Royer, Measurement of the Wigner function. Phys. Rev. Lett. 55 (1985), 2745-2748. MR 861946

[72] N. Seiberg and E. Witten, String theory and noncommutative geometry. J. High Energy Phys. 09 (1999) 032. Zbl 0957.81085 MR 1720697

[73] R. L. Stratonovich, On distributions in representation space. Soviet Phys. JETP 4 (1957), 891-898. Zbl 0082.19302 MR 0088173

[74] N. Tatsuuma, Plancherel formula for non-unimodular locally compact groups. J. Math. Kyoto Univ. 12 (1972), 179-261. Zbl 0241.22017 MR 0299729

[75] A. Unterberger, Calcul de Weyl construit sur un autre relation de commutation que celle d'Heisenberg. Reims 1984, unpublished.

[76] A. Unterberger and J. Unterberger, La série discrète de $\operatorname{SL}(2, \mathbf{R})$ et les opérateurs pseudodifférentiels sur une demi-droite. Ann. Sci. École Norm. Sup. (4) 17 (1984), 83-116. Zbl 0549.35119 MR 744069 
[77] J. C. Várilly and J. M. Gracia-Bondía, The Moyal representation for spin. Ann. Physics 190 (1989), 107-148. Zbl 0652.46028 MR 994048

[78] J. C. Várilly and J. M. Gracia-Bondía, On the ultraviolet behavior of quantum fields over noncommutative manifolds. Internat. J. Modern Phys. A 14 (1999), 1305-1323. Zbl 1045.81550 MR 1688039

[79] V. S. Vladimirov, Y. N. Drozzinov, and B. I. Zavialov, Tauberian theorems for generalized functions. Math. Appl. (Soviet Series) 10, Kluwer Academic Publ., Dordrecht 1988. Zbl 0636.40003 MR 0947960

[80] K. Vogel and H. Risken, Determination of quasiprobability distributions in terms of probability distributions for the rotated quadrature phase. Phys. Rev. A 40 (1989), 2847-2849.

[81] A. Weinstein, Traces and triangles in symmetric symplectic spaces. In Symplectic geometry and quantization (Sanda and Yokohama, 1993), Contemp. Math. 179, Amer. Math. Soc., Providence, RI, 1994, 261-270. Zbl 0820.58024 MR 1319613

[82] N. J. Wildberger, On the Fourier transform of a compact semisimple Lie group. J. Austral. Math. Soc. Ser. A 56 (1994), 64-116. Zbl 0842.22015 MR 1250994

[83] N. J. Wildberger, Characters, bi-modules and representations in Lie group harmonic analysis. In Harmonic analysis and hypergroups (Delhi, 1995), Trends Math., Birkhäuser, Boston 1998, 227-242. Zbl 0888.43005 MR 1616256

Received June 11, 2007; revised February 1, 2008

V. Gayral, Laboratoire de Mathématiques, Université de Reims Champagne-Ardenne, 51687 Reims, France

E-mail: victor.gayral@univ-reims.fr

J. M. Gracia-Bondía, Departamento de Física Teórica, Universidad de Zaragoza, 50009 Zaragoza, Spain, and Departamento de Física, Universidad de Costa Rica, 2060 San José, Costa Rica

J. C. Várilly, Departamento de Matemática, Universidad de Costa Rica, 2060 San José, Costa Rica

E-mail: varilly@cariari.ucr.ac.cr 